\title{
A System of Generalized Mixed Equilibrium Problems and Fixed Point Problems for Pseudocontractive Mappings in Hilbert Spaces
}

\author{
Poom Kumam $^{1}$ and Chaichana Jaiboon ${ }^{2}$ \\ ${ }^{1}$ Department of Mathematics, Faculty of Science, King Mongkut's University of Technology Thonburi, \\ KMUTT, Bangkok 10140, Thailand \\ ${ }^{2}$ Department of Mathematics, Faculty of Liberal Arts, Rajamangala University of Technology Rattanakosin, \\ RMUTR, Bangkok 10100, Thailand
}

Correspondence should be addressed to Chaichana Jaiboon, chaichana.j@rmutr.ac.th

Received 2 April 2010; Accepted 11 June 2010

Academic Editor: A. T. M. Lau

Copyright (c) 2010 P. Kumam and C. Jaiboon. This is an open access article distributed under the Creative Commons Attribution License, which permits unrestricted use, distribution, and reproduction in any medium, provided the original work is properly cited.

We introduce and analyze a new iterative algorithm for finding a common element of the set of fixed points of strict pseudocontractions, the set of common solutions of a system of generalized mixed equilibrium problems, and the set of common solutions of the variational inequalities with inverse-strongly monotone mappings in Hilbert spaces. Furthermore, we prove new strong convergence theorems for a new iterative algorithm under some mild conditions. Finally, we also apply our results for solving convex feasibility problems in Hilbert spaces. The results obtained in this paper improve and extend the corresponding results announced by Qin and Kang (2010) and the previously known results in this area.

\section{Introduction}

Let $H$ be a real Hilbert space with inner product $\langle\cdot, \cdot\rangle$ and norm $\|\cdot\|$ and let $E$ be a nonempty closed convex subset of $H$. We denote weak convergence and strong convergence by notations $\rightarrow$ and $\rightarrow$, respectively. Let $S: E \rightarrow E$ be a mapping. In the sequel, we will use $F(S)$ to denote the set of fixed points of $S$, that is, $F(S)=\{x \in E: S x=x\}$.

Definition 1.1. Let $S: E \rightarrow E$ be a mapping. Then $S$ is called

(1) contraction if there exists a constant $\alpha \in[0,1)$ such that

$$
\|S x-S y\| \leq \alpha\|x-y\|, \quad \forall x, y \in E,
$$


(2) nonexpansive if

$$
\|S x-S y\| \leq\|x-y\|, \quad \forall x, y \in E .
$$

Remark 1.2. It is well known that if $E \subset H$ is nonempty, bounded, closed, and convex and $S$ is a nonexpansive mapping on $E$ then $F(S)$ is nonempty; see, for example, [1].

(3) strongly pseudocontractive with the coefficient $\tau \in(0,1)$ if

$$
\langle S x-S y, x-y\rangle \geq \tau\|x-y\|^{2}, \quad \forall x, y \in E,
$$

(4) strictly pseudocontractive with the coefficient $k \in[0,1)$ if

$$
\|S x-S y\|^{2} \leq\|x-y\|^{2}+k\|(I-S) x-(I-S) y\|^{2}, \quad \forall x, y \in E
$$

for such a case, $S$ is also said to be a $k$-strict pseudocontraction, and if $k=0$, then $S$ is a nonexpansive mapping,

(5) pseudocontractive if

$$
\|S x-S y\|^{2} \leq\|x-y\|^{2}+\|(I-S) x-(I-S) y\|^{2}, \quad \forall x, y \in E .
$$

The class of strict pseudocontractions falls into the one between classes of nonexpansive mappings and pseudocontractions. Within the past several decades, many authors have been devoting to the studies on the existence and convergence of fixed points for strict pseudocontractions.

In 1967, Browder and Petryshyn [2] introduced a convex combination method to study strict pseudocontractions in Hilbert spaces. On the other hand, Marino and Xu [3] and Zhou [4] introduced and researched some iterative scheme for finding a fixed point of a strict pseudocontraction mapping. More precisely, take $k \in(0,1)$ and define a mapping $S_{k}$ by

$$
S_{k} x=k x+(1-k) S x, \quad \forall x \in E,
$$

where $S$ is a strict pseudocontraction. Under appropriate restrictions on $k$, it is proved the mapping $S_{k}$ is nonexpansive. Therefore, the techniques of studying nonexpansive mappings can be applied to study more general strict pseudocontractions.

The domain of the function $\varphi: E \rightarrow \mathcal{R} \cup\{+\infty\}$ is the set

$$
\operatorname{dom} \varphi=\{x \in E: \varphi(x)<+\infty\} .
$$

Let $\varphi: E \rightarrow R \cup\{+\infty\}$ be a proper extended real-valued function and let $\Phi$ be a bifunction of $E \times E$ into $\mathcal{R}$ such that $E \cap \operatorname{dom} \varphi \neq \emptyset$, where $\mathcal{R}$ is the set of real numbers. 
Fixed Point Theory and Applications

There exists the generalized mixed equilibrium problem for finding $x \in E$ such that

$$
\Phi(x, y)+\langle\Psi x, y-x\rangle+\varphi(y)-\varphi(x) \geq 0, \quad \forall y \in E
$$

The set of solutions of $(1.8)$ is denoted by $\operatorname{GMEP}(\Phi, \varphi, \Psi)$, that is,

$$
\operatorname{GMEP}(\Phi, \varphi, \Psi)=\{x \in E: \Phi(x, y)+\langle\Psi x, y-x\rangle+\varphi(y)-\varphi(x) \geq 0, \forall y \in E\}
$$

We see that $x$ is a solution of problem (1.8) implies that $x \in \operatorname{dom} \varphi$.

\section{Special Examples}

(1) If $\Psi=0$, problem (1.8) is reduced into the mixed equilibrium problem for finding $x \in E$ such that

$$
\Phi(x, y)+\varphi(y)-\varphi(x) \geq 0, \quad \forall y \in E
$$

Problem (1.10) was studied by Ceng and Yao [5]. The set of solutions of (1.10) is denoted by $\operatorname{MEP}(\Phi, \varphi)$.

(2) If $\varphi=0$, problem (1.8) is reduced into the generalized equilibrium problem for finding $x \in E$ such that

$$
\Phi(x, y)+\langle\Psi x, y-x\rangle \geq 0, \quad \forall y \in E
$$

Problem (1.11) was studied by Takahashi and Toyoda [6]. The set of solutions of (1.11) is denoted by $\operatorname{GEP}(\Phi, \Psi)$.

(3) If $\Psi=0$ and $\varphi=0$, problem (1.8) is reduced into the equilibrium problem for finding $x \in E$ such that

$$
\Phi(x, y) \geq 0, \quad \forall y \in E
$$

Problem (1.12) was studied by Blum and Oettli [7]. The set of solutions of (1.12) is denoted by $\mathrm{EP}(\Phi)$.

(4) If $\Phi=0$, problem (1.8) is reduced into the mixed variational inequality of Browder type for finding $x \in E$ such that

$$
\langle\Psi x, y-x\rangle+\varphi(y)-\varphi(x) \geq 0, \quad \forall y \in E
$$

Problem (1.13) was studied by Browder [8]. The set of solutions of (1.13) is denoted by $\operatorname{VI}(E, \Psi, \varphi)$.

(5) If $\Phi=0$ and $\varphi=0$, problem (1.8) is reduced into the variational inequality problem for finding $x \in E$ such that

$$
\langle\Psi x, y-x\rangle \geq 0, \quad \forall y \in E
$$


Problem (1.14) was studied by Hartman and Stampacchia [9]. The set of solutions of (1.14) is denoted by $\mathrm{VI}(E, \Psi)$. The variational inequality has been extensively studied in the literature. See, for example, $[7,10,11]$ and the references therein.

(6) If $\Phi=0$ and $\Psi=0$, problem (1.8) is reduced into the minimize problem for finding $x \in E$ such that

$$
\varphi(y)-\varphi(x) \geq 0, \quad \forall y \in E
$$

The set of solutions of (1.15) is denoted by $\operatorname{Argmin}(\varphi)$.

The generalized mixed equilibrium problems include fixed point problems, variational inequality problems, optimization problems, Nash equilibrium problems, and the equilibrium problem as special cases. Numerous problems in physics, optimization, and economics reduce to find a solution of (1.8). In 1997, Combettes and Hirstoaga [12] introduced an iterative scheme of finding the best approximation to initial data when $\operatorname{EP}(\Phi)$ is nonempty and proved a strong convergence theorem. Many authors have proposed some useful methods for solving the $\operatorname{GMEP}(\Phi, \varphi, \Psi), \operatorname{MEP}(\Phi, \varphi)$, and $\operatorname{EP}(\Phi)$; see, for instance, $[5,12-23]$.

Definition 1.3. Let $B: E \rightarrow H$ be a nonlinear mapping. Then $B$ is called

(1) monotone if

$$
\langle B x-B y, x-y\rangle \geq 0, \quad \forall x, y \in E,
$$

(2) $\beta$-strongly monotone if there exists a constant $\beta>0$ such that

$$
\langle B x-B y, x-y\rangle \geq \beta\|x-y\|^{2}, \quad \forall x, y \in E,
$$

(3) $\eta$-Lipschitz continuous if there exists a positive real number $\eta$ such that

$$
\|B x-B y\| \leq \eta\|x-y\|, \quad \forall x, y \in E,
$$

(4) $\beta$-inverse-strongly monotone if there exists a constant $\beta>0$ such that

$$
\langle B x-B y, x-y\rangle \geq \beta\|B x-B y\|^{2}, \quad \forall x, y \in E
$$

Remark 1.4. It is obvious that any $\beta$-inverse-strongly monotone mappings $B$ are monotone and $1 / \beta$-Lipschitz continuous.

For finding a common element of the set of fixed points of a nonexpansive mapping and the set of solution of variational inequalities for a $\beta$-inverse-strongly monotone mapping, Takahashi and Toyoda [6] introduced the following iterative scheme:

$$
\begin{gathered}
x_{0} \in E \text { chosen arbitrary, } \\
x_{n+1}=\alpha_{n} x_{n}+\left(1-\alpha_{n}\right) S P_{E}\left(x_{n}-\lambda_{n} B x_{n}\right), \quad \forall n \geq 0,
\end{gathered}
$$


where $P_{E}$ is the metric projection of $H$ onto $E, B$ is a $\beta$-inverse-strongly monotone mapping, $\left\{\alpha_{n}\right\}$ is a sequence in $(0,1)$, and $\left\{\lambda_{n}\right\}$ is a sequence in $(0,2 \beta)$. They showed that if $F(S) \cap$ $\mathrm{VI}(E, B)$ is nonempty, then the sequence $\left\{x_{n}\right\}$ generated by (1.20) converges weakly to some $q \in F(S) \cap \operatorname{VI}(E, B)$.

On the other hand, Y. Yao and J.-C Yao [24] introduced the following iterative process defined recursively by

$$
\begin{gathered}
x_{1}=x \in E \text { chosen arbitrary, } \\
y_{n}=P_{E}\left(x_{n}-\lambda_{n} B x_{n}\right), \\
x_{n+1}=\alpha_{n} x+\beta_{n} x_{n}+\gamma_{n} S P_{E}\left(y_{n}-\lambda_{n} B y_{n}\right), \quad \forall n \geq 1,
\end{gathered}
$$

where $B$ is a $\beta$-inverse-strongly monotone mapping, $\left\{\alpha_{n}\right\},\left\{\beta_{n}\right\}$, and $\left\{\gamma_{n}\right\}$ are sequences in the interval $[0,1]$, and $\left\{\lambda_{n}\right\}$ is a sequence in $(0,2 \beta)$. They showed that if $F(S) \cap \operatorname{VI}(E, B)$ is nonempty, then the sequence $\left\{x_{n}\right\}$ generated by (1.21) converges strongly to some $q \in F(S) \cap$ $\mathrm{VI}(E, B)$.

Let $A$ be a strongly positive linear bounded operator on $H$ if there is a constant $\bar{\gamma}>0$ with property

$$
\langle A x, x\rangle \geq \bar{\gamma}\|x\|^{2}, \quad \forall x \in H
$$

A typical problem is to minimize a quadratic function over the set of the fixed points a nonexpansive mapping on a real Hilbert space $H$ :

$$
\min _{x \in E} \frac{1}{2}\langle A x, x\rangle-\langle x, b\rangle
$$

where $A$ is a linear bounded operator, $E$ is the fixed point set of a nonexpansive mapping $S$ on $H$, and $b$ is a given point in $H$. Moreover, it is shown in [25] that the sequence $\left\{x_{n}\right\}$ defined by the scheme

$$
x_{n+1}=\epsilon_{n} \gamma f\left(x_{n}\right)+\left(1-\epsilon_{n} A\right) S x_{n}
$$

converges strongly to $q=P_{F(S)}(I-A+\gamma f)(q)$. Recently, Plubtieng and Punpaeng [26] proposed the following iterative algorithm:

$$
\begin{gathered}
\Phi\left(u_{n}, y\right)+\frac{1}{r_{n}}\left\langle y-u_{n}, u_{n}-x_{n}\right\rangle \geq 0, \quad \forall y \in H, \\
x_{n+1}=\epsilon_{n} \gamma f\left(x_{n}\right)+\left(I-\epsilon_{n} A\right) S u_{n} .
\end{gathered}
$$

They proved that if the sequences $\left\{\epsilon_{n}\right\}$ and $\left\{r_{n}\right\}$ of parameters satisfy appropriate condition, then the sequences $\left\{x_{n}\right\}$ and $\left\{u_{n}\right\}$ both converge to the unique solution $q$ of the variational inequality:

$$
\langle(A-\gamma f) q, x-q\rangle \geq 0, \quad \forall x \in F(S) \cap \operatorname{EP}(\Phi),
$$


which is the optimality condition for the minimization problem:

$$
\min _{x \in F(S) \cap \operatorname{EP}(\phi)} \frac{1}{2}\langle A x, x\rangle-h(x),
$$

where $h$ is a potential function for $\gamma f$ (i.e., $h^{\prime}(x)=\gamma f(x)$ for $x \in H$ ).

Very recently, Ceng et al. [27] introduced iterative scheme for finding a common element of the set of solutions of equilibrium problems and the of fixed points of a $k$-strict pseudocontraction mapping defined in the setting of real Hilbert space $H: x_{0} \in H$ and let

$$
\begin{gathered}
\Phi\left(u_{n}, y\right)+\frac{1}{r_{n}}\left\langle y-u_{n}, u_{n}-x_{n}\right\rangle \geq 0, \quad \forall y \in E \\
x_{n+1}=\alpha_{n} u_{n}+\left(1-\alpha_{n}\right) S u_{n}
\end{gathered}
$$

where $\left\{\alpha_{n}\right\} \subset[a, b]$ for some $a, b \in(k, 1)$ and $\left\{r_{n}\right\} \subset(0, \infty)$ satisfies $\liminf _{n \rightarrow \infty} r_{n}>0$. Further, they proved that $\left\{x_{n}\right\}$ and $\left\{u_{n}\right\}$ converge weakly to $q \in F(S) \cap \operatorname{EP}(\Phi)$, where $q=P_{F(S) \cap \operatorname{EP}(\Phi)} x_{0}$.

On the other hand, for finding a common element of the set of fixed points of a $k$ strict pseudocontraction mapping and the set of solutions of an equilibrium problems in a real Hilbert space, Liu [28] introduced the following iterative scheme:

$$
\begin{gathered}
\Phi\left(u_{n}, y\right)+\frac{1}{r_{n}}\left\langle y-u_{n}, u_{n}-x_{n}\right\rangle \geq 0, \quad \forall y \in E, \\
y_{n}=\beta_{n} u_{n}+\left(1-\beta_{n}\right) S u_{n}, \\
x_{n+1}=\epsilon_{n} \gamma f\left(x_{n}\right)+\left(I-\epsilon_{n} A\right) y_{n}, \quad \forall n \geq 1,
\end{gathered}
$$

where $S$ is a $k$-strict pseudocontraction mapping and $\left\{\epsilon_{n}\right\}$ and $\left\{\beta_{n}\right\}$ are sequences in $[0,1]$. They proved that under certain appropriate conditions over $\left\{\epsilon_{n}\right\},\left\{\beta_{n}\right\}$, and $\left\{r_{n}\right\}$, the sequences $\left\{x_{n}\right\}$ and $\left\{u_{n}\right\}$ both converge strongly to some $q \in F(S) \cap \operatorname{EP}(\Phi)$, which solves some variational inequality problems (1.26). 
In 2008, Ceng and Yao [5] introduced an iterative scheme for finding a common fixed point of a finite family of nonexpansive mappings and the set of solutions of a problem (1.8) in Hilbert spaces and obtained the strong convergence theorem which used the following condition.

(G) $K: E \rightarrow \mathcal{R}$ is $\eta$-strongly convex with constant $\sigma>0$ and its derivative $K^{\prime}$ is sequentially continuous from the weak topology to the strong topology. We note that the condition $(G)$ for the function $K: E \rightarrow R$ is a very strong condition. We also note that the condition $(G)$ does not cover the case $K(x)=\|x\|^{2} / 2$ and $\eta(x, y)=x-y$ for each $(x, y) \in E \times E$. Very recently, Wangkeeree and Wangkeeree [29] introduced a general iterative method for finding a common element of the set of solutions of the mixed equilibrium problems, the set of fixed point of a $k$-strict pseudocontraction mapping, and the set of solutions of the variational inequality for an inverse-strongly monotone mapping in Hilbert spaces. They obtained a strong convergence theorem except the condition $(G)$ for the sequences generated by these processes.

In 2009, Qin and Kang [30] introduced an explicit viscosity approximation method for finding a common element of the set of fixed points of strict pseudocontraction and the set of solutions of variational inequalities with inverse-strongly monotone mappings in Hilbert spaces. Let $\left\{x_{n}\right\}$ be a sequence generated by the following iterative algorithm:

$$
\begin{gathered}
x_{1} \in E, \\
z_{n}=P_{E}\left(x_{n}-\mu_{n} C x_{n}\right), \\
y_{n}=P_{E}\left(x_{n}-\lambda_{n} B x_{n}\right), \\
x_{n+1}=\epsilon_{n} f\left(x_{n}\right)+\beta_{n} x_{n}+\gamma_{n}\left[\alpha_{n}^{(1)} S_{k} x_{n}+\alpha_{n}^{(2)} y_{n}+\alpha_{n}^{(3)} z_{n}\right], \quad \forall n \geq 1 .
\end{gathered}
$$

Then, they proved that under certain appropriate conditions imposed on $\left\{\epsilon_{n}\right\},\left\{\beta_{n}\right\},\left\{\gamma_{n}\right\}$, $\left\{\alpha_{n}^{(1)}\right\},\left\{\alpha_{n}^{(2)}\right\}$, and $\left\{\alpha_{n}^{(3)}\right\}$, the sequence $\left\{x_{n}\right\}$ generated by (1.30) converges strongly to $q \in$ $F(S) \cap \operatorname{VI}(E, B) \cap \operatorname{VI}(E, C)$, where $q=P_{F(S) \cap V I}(E, B) \cap V I(E, C) f(q)$.

In the present paper, motivated and inspired by Qin and Kang [30], Peng and Yao [21], Plubtieng and Punpaeng [26], and Liu [28], we introduce a new general iterative scheme for finding a common element of the set of fixed points of strict pseudocontractions, the set of common solutions of the system of generalized mixed equilibrium problems, and the set of common solutions of the variational inequalities for inverse-strongly monotone mappings in Hilbert spaces. We obtain a strong convergence theorem for the sequences generated by these processes under some parameter controlling conditions. The results in this paper extend and improve the corresponding recent results of Qin and Kang [30], Peng and Yao [21], Plubtieng and Punpaeng [26], and Liu [28] and many others.

\section{Preliminaries}

Let $H$ be a real Hilbert space and let $E$ be a nonempty closed convex subset of $H$. In a real Hilbert space $H$, it is well known that

$$
\|\lambda x+(1-\lambda) y\|^{2}=\lambda\|x\|^{2}+(1-\lambda)\|y\|^{2}-\lambda(1-\lambda)\|x-y\|^{2}, \quad \forall \lambda \in[0,1], \forall x, y \in H
$$


For any $x \in H$, there exists a unique nearest point in $E$, denoted by $P_{E} x$, such that

$$
\left\|x-P_{E} x\right\| \leq\|x-y\|, \quad \forall y \in E
$$

The mapping $P_{E}$ is called the metric projection of $H$ onto $E$.

It is well known that $P_{E}$ is a firmly nonexpansive mapping of $H$ onto $E$, that is,

$$
\left\langle x-y, P_{E} x-P_{E} y\right\rangle \geq\left\|P_{E} x-P_{E} y\right\|^{2}, \quad \forall x, y \in H
$$

Moreover, $P_{E} x$ is characterized by the following properties: $P_{E} x \in E$ and

$$
\begin{gathered}
\left\langle x-P_{E} x, y-P_{E} x\right\rangle \leq 0, \\
\|x-y\|^{2} \geq\left\|x-P_{E} x\right\|^{2}+\left\|y-P_{E} x\right\|^{2}
\end{gathered}
$$

for all $x \in H, y \in E$.

Lemma 2.1. Let $E$ be a nonempty closed convex subset of a real Hilbert space $H$. Given $x \in H$ and $z \in E$, then,

$$
z=P_{E} x \Longleftrightarrow\langle x-z, y-z\rangle \leq 0, \quad \forall y \in E
$$

Lemma 2.2. Let $H$ be a Hilbert space, let $E$ be a nonempty closed convex subset of $H$, and let $B$ be a mapping of $E$ into $H$. Let $u \in E$. Then for $\lambda>0$,

$$
u \in V I(E, B) \Longleftrightarrow u=P_{E}(u-\lambda B u)
$$

where $P_{E}$ is the metric projection of $H$ onto $E$.

A set-valued mapping $T: H \rightarrow 2^{H}$ is called a monotone if for all $x, y \in H, f \in T x$ and $g \in T y$ imply $\langle x-y, f-g\rangle \geq 0$. A monotone mapping $T: H \rightarrow 2^{H}$ is called maximal if the graph $G(T)$ of $T$ is not properly contained in the graph of any other monotone mapping. It is known that a monotone mapping $T$ is maximal if and only if for $(x, f) \in H \times H,\langle x-y, f-g\rangle \geq$ 0 for every $(y, g) \in G(T)$ implies $f \in T x$. Let $B$ be a monotone map of $E$ into $H, \eta$-Lipschitz continuous mappings and let $N_{E} v$ be the normal cone to $E$ when $v \in E$, that is,

$$
N_{E} v=\{w \in H:\langle v-u, w\rangle \geq 0, \forall u \in E\},
$$

and define a mapping $T$ on $E$ by

$$
T v= \begin{cases}B v+N_{E} v, & v \in E, \\ \emptyset, & v \notin E .\end{cases}
$$

Then $T$ is the maximal monotone and $0 \in T v$ if and only if $v \in \operatorname{VI}(E, B)$; see [31]. 
Lemma 2.3. Let $H$ be a Hilbert space, let $E$ be a nonempty closed convex subset of $H$, and let $\Psi$ : $E \rightarrow H$ be $\rho$-inverse-strongly monotone. It $0<r \leq 2 \rho$, then $I-\rho \Psi$ is a nonexpansive mapping in $H$.

Proof. For all $x, y \in E$ and $0<r \leq 2 \rho$, we have

$$
\begin{aligned}
\|(I-r \Psi) x-(I-r \Psi) y\|^{2} & =\|(x-y)-r(\Psi x-\Psi y)\|^{2} \\
& =\|x-y\|^{2}-2 r\langle x-y, \Psi x-\Psi y\rangle+r^{2}\|\Psi x-\Psi y\|^{2} \\
& \leq\|x-y\|^{2}-2 r \rho\|\Psi x-\Psi y\|+r^{2}\|\Psi x-\Psi y\|^{2} \\
& =\|x-y\|^{2}+r(r-2 \rho)\|\Psi x-\Psi y\|^{2} \\
& \leq\|x-y\|^{2} .
\end{aligned}
$$

So, $I-\rho \Psi$ is a nonexpansive mapping of $E$ into $H$.

Lemma 2.4 (see [32]). Let $(E,\langle\cdot, \cdot\rangle)$ be an inner product space. Then, for all $x, y, z \in E$ and $\alpha, \beta, \gamma \in$ $[0,1]$ with $\alpha+\beta+\gamma=1$, one has

$$
\|\alpha x+\beta y+\gamma z\|^{2}=\alpha\|x\|^{2}+\beta\|y\|^{2}+\gamma\|z\|^{2}-\alpha \beta\|x-y\|^{2}-\alpha \gamma\|x-z\|^{2}-\beta \gamma\|y-z\|^{2} .
$$

Lemma 2.5 (see [25]). Let $E$ be a nonempty closed convex subset of $H$, let $f$ be a contraction of $H$ into itself with $\alpha \in[0,1)$, and let $A$ be a strongly positive linear bounded operator on $H$ with coefficient $\bar{\gamma}>0$. Then, for $0<\gamma<\bar{\gamma} / \alpha$,

$$
\langle x-y,(A-\gamma f) x-(A-\gamma f) y\rangle \geq(\bar{\gamma}-\gamma \alpha)\|x-y\|^{2}, \quad x, y \in H .
$$

That is, $A-\gamma f$ is strongly monotone with coefficient $\bar{\gamma}-\gamma \alpha$.

Lemma 2.6 (see [25]). Assume that $A$ is a strongly positive linear bounded operator on $H$ with coefficient $\bar{\gamma}>0$ and $0<\vartheta \leq\|A\|^{-1}$. Then $\|I-\vartheta A\| \leq 1-\vartheta \bar{\gamma}$.

Lemma 2.7 (see [4]). Let $E$ be a nonempty closed convex subset of a real Hilbert space $H$ and let $S: E \rightarrow E$ be a $k$-strict pseudocontraction mapping with a fixed point. Then $F(S)$ is closed and convex. Define $S_{k}: E \rightarrow E$ by $S_{k}=k x+(1-k) S x$ for each $x \in E$. Then $S_{k}$ is nonexpansive such that $F\left(S_{k}\right)=F(S)$.

Lemma 2.8 (see [33]). Let $E$ be a closed convex subset of a Hilbert space $H$ and let $S: E \rightarrow E$ be a nonexpansive mapping. Then $I-S$ is demiclosed at zero, that is,

$$
x_{n} \rightarrow x, \quad x_{n}-S x_{n} \longrightarrow 0 \quad \text { implies } x=S x
$$

Lemma 2.9 (see [34]). Let $E$ be a nonempty closed convex subset of a strictly convex Banach space $X$. Let $\left\{T_{n}: n \in \mathbb{N}\right\}$ be a sequence of nonexpansive mappings on E. Suppose that $\bigcap_{n=1}^{\infty} F\left(T_{n}\right)$ is 
nonempty. Let $\delta_{n}$ be a sequence of positive number with $\sum_{n=1}^{\infty} \delta_{n}=1$. Then a mapping $S$ on $E$ defined by

$$
S x=\sum_{n=1}^{\infty} \delta_{n} T_{n} x
$$

for $x \in E$ is well defined and nonexpansive and $F(S)=\bigcap_{n=1}^{\infty} F\left(T_{n}\right)$ holds.

For solving the mixed equilibrium problem, let us give the following assumptions for the bifunction $\Phi$, the function $\varphi$, and the set $E$ :

(A1) $\Phi(x, x)=0$ for all $x \in E$;

(A2) $\Phi$ is monotone, that is, $\Phi(x, y)+\Phi(y, x) \leq 0$ for all $x, y \in E$;

(A3) for each $x, y, z \in E, \lim _{t \rightarrow 0} \Phi(t z+(1-t) x, y) \leq \Phi(x, y)$;

(A4) for each $x \in E, y \mapsto \Phi(x, y)$ is convex and lower semicontinuous;

(A5) for each $y \in E, x \mapsto \Phi(x, y)$ is weakly upper semicontinuous;

(B1) for each $x \in H$ and $r>0$, there exists a bounded subset $D_{x} \subseteq E$ and $y_{x} \in E$ such that for any $z \in E \backslash D_{x}$,

$$
\Phi\left(z, y_{x}\right)+\varphi\left(y_{x}\right)-\varphi(z)+\frac{1}{r}\left\langle y_{x}-z, z-x\right\rangle<0
$$

(B2) $E$ is a bounded set.

By similar argument as in the proof of Lemma 2.10 in [35], we have the following lemma appearing.

Lemma 2.10. Let $E$ be a nonempty closed convex subset of $H$. Let $\Phi: E \times E \rightarrow R$ be a bifunction satisfies (A1)-(A5) and let $\varphi: E \rightarrow \mathcal{R} \cup\{+\infty\}$ be a proper lower semicontinuous and convex function. Assume that either (B1) or (B2) holds. For $r>0$ and $x \in H$, define a mapping $T_{r}^{\Phi}: H \rightarrow E$ as follows:

$$
T_{r}^{\Phi}(x)=\left\{z \in E: \Phi(z, y)+\varphi(y)-\varphi(z)+\frac{1}{r}\langle y-z, z-x\rangle \geq 0, \forall y \in E\right\}
$$

for all $z \in H$. Then, the following holds:

(i) for each $x \in H, T_{r}^{\Phi}(x) \neq \emptyset$;

(ii) $T_{r}^{\Phi}$ is single-valued;

(iii) $T_{r}^{\Phi}$ is firmly nonexpansive, that is, for any $x, y \in H$,

$$
\left\|T_{r}^{\Phi} x-T_{r}^{\Phi} y\right\|^{2} \leq\left\langle T_{r}^{\Phi} x-T_{r}^{\Phi} y, x-y\right\rangle
$$

(iv) $F\left(T_{r}^{\Phi}\right)=\operatorname{MEP}(\Phi, \varphi)$;

(v) $\operatorname{MEP}(\Phi, \varphi)$ is closed and convex. 
Remark 2.11. We remark that Lemma 2.10 is not a consequence of Lemma 3.1 in [5], because the condition of the sequential continuity from the weak topology to the strong topology for the derivative $K^{\prime}$ of the function $K: E \rightarrow \mathcal{R}$ does not cover the case $K(x)=\|x\|^{2} / 2$.

Lemma 2.12 (see [36]). Let $\left\{x_{n}\right\}$ and $\left\{l_{n}\right\}$ be bounded sequences in a Banach space $X$ and let $\left\{\beta_{n}\right\}$ be a sequence in $[0,1]$ with $0<\lim _{\inf _{n \rightarrow \infty}} \beta_{n} \leq \lim \sup _{n \rightarrow \infty} \beta_{n}<1$. Suppose $x_{n+1}=\left(1-\beta_{n}\right) l_{n}+\beta_{n} x_{n}$ for all integers $n \geq 1$ and $\lim \sup _{n \rightarrow \infty}\left(\left\|l_{n+1}-l_{n}\right\|-\left\|x_{n+1}-x_{n}\right\|\right) \leq 0$. Then, $\lim _{n \rightarrow \infty}\left\|l_{n}-x_{n}\right\|=0$.

Lemma 2.13 (see [37]). Assume that $\left\{a_{n}\right\}$ is a sequence of nonnegative real numbers such that

$$
a_{n+1} \leq\left(1-\rho_{n}\right) a_{n}+\sigma_{n}, \quad n \geq 1
$$

where $\left\{\varrho_{n}\right\}$ is a sequence in $(0,1)$ and $\left\{\sigma_{n}\right\}$ is a sequence in $R$ such that

(1) $\sum_{n=1}^{\infty} Q_{n}=\infty$,

(2) $\lim \sup _{n \rightarrow \infty}\left(\sigma_{n} / \varphi_{n}\right) \leq 0$ or $\sum_{n=1}^{\infty}\left|\sigma_{n}\right|<\infty$.

Then $\lim _{n \rightarrow \infty} a_{n}=0$.

Lemma 2.14. Let $H$ be a real Hilbert space. Then for all $x, y \in H$,

$$
\|x+y\|^{2} \leq\|x\|^{2}+2\langle y, x+y\rangle .
$$

\section{Main Results}

In this section, we will use the new approximation iterative method to prove a strong convergence theorem for finding a common element of the set of fixed points of strict pseudocontractions, the set of common solutions of the system of generalized mixed equilibrium problems, and the set of a common solutions of the variational inequalities for inverse-strongly monotone mappings in a real Hilbert space.

Theorem 3.1. Let $E$ be a nonempty closed convex subset of a real Hilbert space $H$. Let $\Phi_{1}$ and $\Phi_{2}$ be two bifunctions from $E \times E$ to $R$ satisfying $(A 1)-(A 5)$ and let $\varphi: E \rightarrow \mathcal{R} \cup\{+\infty\}$ be a proper lower semicontinuous and convex function with either (B1) or (B2). Let $C: E \rightarrow H$ be a $\xi$-inverse-strongly monotone mapping, let $\Psi_{1}: E \rightarrow H$ be a $\rho$-inverse-strongly monotone mapping, let $\Psi_{2}: E \rightarrow H$ be an $\omega$-inverse-strongly monotone mapping, and let $B: E \rightarrow H$ be a $\beta$-inverse-strongly monotone mapping. Let $f: E \rightarrow E$ be an $\alpha$-contraction with coefficient $\alpha(0 \leq \alpha<1)$ and let $A$ be a strongly positive linear bounded operator on $H$ with coefficient $\bar{\gamma}>0$ and $0<\gamma<\bar{\gamma} / \alpha$. Let $S: E \rightarrow E$ be a $k$-strict pseudocontraction with a fixed point. Define a mapping $S_{k}: E \rightarrow E$ by $S_{k} x=k x+(1-k) S x$, for all $x \in E$. Suppose that

$$
\Theta:=F(S) \cap V I(E, B) \cap V I(E, C) \cap \operatorname{GMEP}\left(\Phi_{1}, \varphi, \Psi_{1}\right) \cap \operatorname{GMEP}\left(\Phi_{2}, \varphi, \Psi_{2}\right) \neq \emptyset
$$


Let $\left\{x_{n}\right\}$ be a sequence generated by the following iterative algorithm:

$$
\begin{gathered}
x_{1} \in E, \quad u_{n} \in E, \quad v_{n} \in E, \\
\Phi_{1}\left(u_{n}, u\right)+\varphi(x)-\varphi\left(u_{n}\right)+\left\langle\Psi_{1} x_{n}, u-u_{n}\right\rangle+\frac{1}{r}\left\langle u-u_{n}, u_{n}-x_{n}\right\rangle \geq 0, \quad \forall u \in E, \\
\Phi_{2}\left(v_{n}, v\right)+\varphi(x)-\varphi\left(v_{n}\right)+\left\langle\Psi_{2} x_{n}, v-v_{n}\right\rangle+\frac{1}{s}\left\langle v-v_{n}, v_{n}-x_{n}\right\rangle \geq 0, \quad \forall v \in E, \\
k_{n}=\alpha_{n}^{(1)} S_{k} x_{n}+\alpha_{n}^{(2)} P_{E}\left(x_{n}-\lambda_{n} B x_{n}\right)+\alpha_{n}^{(3)} P_{E}\left(x_{n}-\mu_{n} C x_{n}\right)+\alpha_{n}^{(4)} u_{n}+\alpha_{n}^{(5)} v_{n}, \\
x_{n+1}=\epsilon_{n} \gamma f\left(x_{n}\right)+\beta_{n} x_{n}+\left(\left(1-\beta_{n}\right) I-\epsilon_{n} A\right) k_{n}, \quad \forall n \geq 1,
\end{gathered}
$$

where $\left\{\epsilon_{n}\right\},\left\{\beta_{n}\right\},\left\{\gamma_{n}\right\}$, and $\left\{\alpha_{n}^{(i)}\right\}$ are sequences in $(0,1)$, where $i=1,2,3,4,5, r \in(0,2 \rho), s \in$ $(0,2 \omega)$, and $\left\{\lambda_{n}\right\}$ and $\left\{\mu_{n}\right\}$ are positive sequences. Assume that the control sequences satisfy the following restrictions:

(C1) $\sum_{i=1}^{5} \alpha_{n}^{(i)}=1$,

(C2) $\lim _{n \rightarrow \infty} \epsilon_{n}=0$ and $\sum_{n=1}^{\infty} \epsilon_{n}=\infty$,

(C3) $0<\liminf _{n \rightarrow \infty} \beta_{n} \leq \lim \sup _{n \rightarrow \infty} \beta_{n}<1$,

(C4) $\lim _{n \rightarrow \infty}\left|\lambda_{n+1}-\lambda_{n}\right|=\lim _{n \rightarrow \infty}\left|\mu_{n+1}-\mu_{n}\right|=0$,

(C5) $d \leq \lambda_{n} \leq 2 \beta$ and $e \leq \mu_{n} \leq 2 \xi$, where $d$, e are two positive constants,

(C6) $\lim _{n \rightarrow \infty} \alpha_{n}^{(i)}=\alpha^{(i)} \in(0,1)$, where $i=1,2,3,4,5$.

Then, $\left\{x_{n}\right\}$ converges strongly to a point $q \in \Theta$ which is the unique solution of the variational inequality:

$$
\langle(A-\gamma f) q, x-q\rangle \geq 0, \quad \forall x \in \Theta .
$$

Equivalently, one has $q=P_{\Theta}(I-A+\gamma f)(q)$.

Proof. Since $\epsilon_{n} \rightarrow 0$, as $n \rightarrow \infty$, we may assume, without loss of generality, that $\epsilon_{n} \leq(1-$ $\left.\beta_{n}\right)\|A\|^{-1}$ for all $n \in \mathbb{N}$. By Lemma 2.6, we know that if $0 \leq \vartheta \leq\|A\|^{-1}$, then $\|I-\vartheta A\| \leq 1-\vartheta \bar{\gamma}$. We will assume that $\|I-A\| \leq 1-\bar{\gamma}$. Since $A$ is a strongly positive bounded linear operator on $H$, we have

$$
\|A\|=\sup \{|\langle A x, x\rangle|: x \in H,\|x\|=1\} .
$$

Observe that

$$
\begin{aligned}
\left\langle\left(\left(1-\beta_{n}\right) I-\epsilon_{n} A\right) x, x\right\rangle & =1-\beta_{n}-\epsilon_{n}\langle A x, x\rangle \\
& \geq 1-\beta_{n}-\epsilon_{n}\|A\| \\
& \geq 0,
\end{aligned}
$$


and so this shows that $\left(1-\beta_{n}\right) I-\epsilon_{n} A$ is positive. It follows that

$$
\begin{aligned}
\left\|\left(1-\beta_{n}\right) I-\epsilon_{n} A\right\| & =\sup \left\{\left|\left\langle\left(\left(1-\beta_{n}\right) I-\epsilon_{n} A\right) x, x\right\rangle\right|: x \in H,\|x\|=1\right\} \\
& =\sup \left\{1-\beta_{n}-\epsilon_{n}\langle A x, x\rangle: x \in H,\|x\|=1\right\} \\
& \leq 1-\beta_{n}-\epsilon_{n} \bar{\gamma} .
\end{aligned}
$$

Since $f$ is a contraction of $H$ into itself with $\alpha \in[0,1)$, then, we have

$$
\begin{aligned}
\left\|P_{\Theta}(I-A+\gamma f)(x)-P_{\Theta}(I-A+\gamma f)(y)\right\| & \leq\|(I-A+\gamma f)(x)-(I-A+\gamma f)(y)\| \\
& \leq\|I-A\|\|x-y\|+\gamma\|f(x)-f(y)\| \\
& \leq(1-\bar{\gamma})\|x-y\|+\gamma \alpha\|x-y\| \\
& =(1-(\bar{\gamma}-\gamma \alpha))\|x-y\|, \quad \forall x, y \in H .
\end{aligned}
$$

Since $0<1-(\bar{\gamma}-\gamma \alpha)<1$, it follows that $P_{\Theta}(I-A+\gamma f)$ is a contraction of $H$ into itself. Therefore the Banach Contraction Mapping Principle implies that there exists a unique element $q \in H$ such that $q=P_{\Theta}(I-A+\gamma f)(q)$.

Next, we will divide the proof into five steps.

Step 1. We claim that $\left\{x_{n}\right\}$ is bounded.

Indeed, let $p \in \Theta$ and by Lemma 2.10, we obtain

$$
p=P_{E}\left(p-\lambda_{n} B p\right)=P_{E}\left(p-\mu_{n} C p\right)=T_{r}^{\Phi_{1}}\left(I-r \Psi_{1}\right) p=T_{s}^{\Phi_{2}}\left(I-s \Psi_{2}\right) p
$$

Note that $u_{n}=T_{r}^{\Phi_{1}}\left(I-r \Psi_{1}\right) x_{n} \in \operatorname{dom} \varphi$ and $v_{n}=T_{s}^{\Phi_{2}}\left(I-s \Psi_{2}\right) x_{n} \in$ dom $\varphi$; we have

$$
\begin{aligned}
& \left\|u_{n}-p\right\|=\left\|T_{r}^{\Phi_{1}}\left(I-r \Psi_{1}\right) x_{n}-T_{r}^{\Phi_{1}}\left(I-r \Psi_{1}\right) p\right\| \leq\left\|x_{n}-p\right\|, \\
& \left\|v_{n}-p\right\|=\left\|T_{s}^{\Phi_{2}}\left(I-s \Psi_{2}\right) x_{n}-T_{s}^{\Phi_{2}}\left(I-s \Psi_{2}\right) p\right\| \leq\left\|x_{n}-p\right\| .
\end{aligned}
$$


Put $z_{n}=P_{E}\left(x_{n}-\mu_{n} C x_{n}\right)$ and $y_{n}=P_{E}\left(x_{n}-\lambda_{n} B x_{n}\right)$. For each $\lambda_{n} \leq 2 \beta$ and $\mu_{n} \leq 2 \xi$ by Lemma 2.3, we get that $I-\lambda_{n} B$ and $I-\mu_{n} B$ are nonexpansive. Thus, we have

$$
\begin{aligned}
\left\|z_{n}-p\right\| & =\left\|P_{E}\left(x_{n}-\mu_{n} C x_{n}\right)-P_{E}\left(p-\mu_{n} C p\right)\right\| \\
& \leq\left\|\left(x_{n}-\mu_{n} C x_{n}\right)-\left(p-\mu_{n} C p\right)\right\| \\
& =\left\|\left(I-\mu_{n} C\right) x_{n}-\left(I-\mu_{n} C\right) p\right\| \\
& \leq\left\|x_{n}-p\right\|, \\
\left\|y_{n}-p\right\| & =\left\|P_{E}\left(x_{n}-\lambda_{n} B x_{n}\right)-P_{E}\left(p-\lambda_{n} B p\right)\right\| \\
& \leq\left\|\left(x_{n}-\lambda_{n} B x_{n}\right)-\left(p-\lambda_{n} B p\right)\right\| \\
& =\left\|\left(I-\lambda_{n} B\right) v_{n}-\left(I-\lambda_{n} B\right) p\right\| \\
& \leq\left\|v_{n}-p\right\| \leq\left\|x_{n}-p\right\| .
\end{aligned}
$$

From Lemma 2.7, we have that $S_{k}$ is nonexpansive with $F\left(S_{k}\right)=F(S)$. It follows that

$$
\begin{aligned}
\left\|k_{n}-p\right\| & =\left\|\alpha_{n}^{(1)} S_{k} x_{n}+\alpha_{n}^{(2)} y_{n}+\alpha_{n}^{(3)} z_{n}+\alpha_{n}^{(4)} u_{n}+\alpha_{n}^{(5)} v_{n}\right\| \\
& \leq \alpha_{n}^{(1)}\left\|S_{k} x_{n}-p\right\|+\alpha_{n}^{(2)}\left\|y_{n}-p\right\|+\alpha_{n}^{(3)}\left\|z_{n}-p\right\|+\alpha_{n}^{(3)}\left\|u_{n}-p\right\|+\alpha_{n}^{(3)}\left\|v_{n}-p\right\| \\
& \leq \alpha_{n}^{(1)}\left\|x_{n}-p\right\|+\alpha_{n}^{(2)}\left\|x_{n}-p\right\|+\alpha_{n}^{(3)}\left\|x_{n}-p\right\|+\alpha_{n}^{(3)}\left\|x_{n}-p\right\|+\alpha_{n}^{(3)}\left\|x_{n}-p\right\| \\
& =\left\|x_{n}-p\right\|
\end{aligned}
$$

which yields that

$$
\begin{aligned}
\left\|x_{n+1}-p\right\| & =\left\|\epsilon_{n}\left(\gamma f\left(x_{n}\right)-A p\right)+\beta_{n}\left(x_{n}-p\right)+\left(\left(1-\beta_{n}\right) I-\epsilon_{n} A\right)\left(k_{n}-p\right)\right\| \\
& \leq\left(1-\beta_{n}-\epsilon_{n} \bar{\gamma}\right)\left\|k_{n}-p\right\|+\beta_{n}\left\|x_{n}-p\right\|+\epsilon_{n}\left\|\gamma f\left(x_{n}\right)-A p\right\| \\
& \leq\left(1-\beta_{n}-\epsilon_{n} \bar{\gamma}\right)\left\|x_{n}-p\right\|+\beta_{n}\left\|x_{n}-p\right\|+\epsilon_{n}\left\|\gamma f\left(x_{n}\right)-A p\right\| \\
& \leq\left(1-\epsilon_{n} \bar{\gamma}\right)\left\|x_{n}-p\right\|+\epsilon_{n} \gamma\left\|f\left(x_{n}\right)-f(p)\right\|+\epsilon_{n}\|\gamma f(p)-A p\|
\end{aligned}
$$


Fixed Point Theory and Applications

$$
\begin{aligned}
& \leq\left(1-\epsilon_{n} \bar{\gamma}\right)\left\|x_{n}-p\right\|+\epsilon_{n} \gamma \alpha\left\|x_{n}-p\right\|+\epsilon_{n}\|\gamma f(p)-A p\| \\
& =\left(1-(\bar{\gamma}-\alpha \gamma) \epsilon_{n}\right)\left\|x_{n}-p\right\|+(\bar{\gamma}-\alpha \gamma) \epsilon_{n} \frac{\|\gamma f(p)-A p\|}{\bar{\gamma}-\alpha \gamma} \\
& \leq \max \left\{\left\|x_{n}-p\right\|, \frac{\|\gamma f(p)-A p\|}{\bar{\gamma}-\alpha \gamma}\right\} \\
& \leq \quad \vdots \\
& \leq \max \left\{\left\|x_{1}-p\right\|, \frac{\|\gamma f(p)-A p\|}{\bar{\gamma}-\alpha \gamma}\right\}, \quad \forall n \in \mathbb{N} .
\end{aligned}
$$

Hence, $\left\{x_{n}\right\}$ is bounded, and so are $\left\{u_{n}\right\},\left\{v_{n}\right\},\left\{z_{n}\right\},\left\{y_{n}\right\},\left\{k_{n}\right\},\left\{f\left(x_{n}\right)\right\},\left\{C x_{n}\right\}$, and $\left\{B x_{n}\right\}$.

Step 2. We claim that $\lim _{n \rightarrow \infty}\left\|x_{n+1}-x_{n}\right\|=0$ and $\lim _{n \rightarrow \infty}\left\|k_{n}-x_{n}\right\|=0$.

Observing that $u_{n}=T_{r}^{\Phi_{1}}\left(I-r \Psi_{1}\right) x_{n} \in \operatorname{dom} \varphi$ and $u_{n+1}=T_{r}^{\Phi_{1}}\left(I-r \Psi_{1}\right) x_{n+1} \in \operatorname{dom} \varphi$, by the nonexpansiveness of $T_{r}^{\Phi_{1}}$, we get

$$
\left\|u_{n+1}-u_{n}\right\|=\left\|T_{r}^{\Phi_{1}}\left(I-r \Psi_{1}\right) x_{n+1}-T_{r}^{\Phi_{1}}\left(I-r \Psi_{1}\right) x_{n}\right\| \leq\left\|x_{n+1}-x_{n}\right\|
$$

Similarly, let $v_{n}=T_{s}^{\Phi_{2}}\left(I-s \Psi_{2}\right) x_{n} \in \operatorname{dom} \varphi$ and $v_{n+1}=T_{s}^{\Phi_{2}}\left(I-s \Psi_{2}\right) x_{n+1} \in \operatorname{dom} \varphi$; we have

$$
\left\|v_{n+1}-v_{n}\right\|=\left\|T_{s}^{\Phi_{2}}\left(I-s \Psi_{2}\right) x_{n+1}-T_{s}^{\Phi_{2}}\left(I-s \Psi_{2}\right) x_{n}\right\| \leq\left\|x_{n+1}-x_{n}\right\|
$$

From $z_{n}=P_{E}\left(x_{n}-\mu_{n} C x_{n}\right)$ and $y_{n}=P_{E}\left(x_{n}-\lambda_{n} B x_{n}\right)$; thus, we compute

$$
\begin{aligned}
\left\|z_{n+1}-z_{n}\right\| & =\left\|P_{E}\left(x_{n+1}-\mu_{n+1} C x_{n+1}\right)-P_{E}\left(x_{n}-\mu_{n} C x_{n}\right)\right\| \| \\
& \leq\left\|\left(x_{n+1}-\mu_{n+1} C x_{n+1}\right)-\left(x_{n}-\mu_{n} C x_{n}\right)\right\| \\
& =\left\|\left(x_{n+1}-\mu_{n+1} C x_{n+1}\right)-\left(x_{n}-\mu_{n+1} C x_{n}\right)+\left(\mu_{n}-\mu_{n+1}\right) C x_{n}\right\| \\
& \leq\left\|\left(x_{n+1}-\mu_{n+1} C x_{n+1}\right)-\left(x_{n}-\mu_{n+1} C x_{n}\right)\right\|+\left|\mu_{n}-\mu_{n+1}\right|\left\|C x_{n}\right\| \\
& =\left\|\left(I-\mu_{n+1} C\right) x_{n+1}-\left(I-\mu_{n+1} C\right) x_{n}\right\|+\left|\mu_{n}-\mu_{n+1}\right|\left\|C x_{n}\right\| \\
& \leq\left\|x_{n+1}-x_{n}\right\|+\left|\mu_{n}-\mu_{n+1}\right|\left\|C x_{n}\right\| .
\end{aligned}
$$

Similarly, we have

$$
\begin{aligned}
\left\|y_{n+1}-y_{n}\right\| & =\left\|P_{E}\left(x_{n+1}-\lambda_{n+1} B x_{n+1}\right)-P_{E}\left(x_{n}-\lambda_{n} B x_{n}\right)\right\| \\
& \leq\left\|x_{n+1}-x_{n}\right\|+\left|\lambda_{n}-\lambda_{n+1}\right|\left\|B x_{n}\right\| .
\end{aligned}
$$


Also noticing that

$$
\begin{gathered}
k_{n}=\alpha_{n}^{(1)} S_{k} x_{n}+\alpha_{n}^{(2)} y_{n}+\alpha_{n}^{(3)} z_{n}+\alpha_{n}^{(4)} u_{n}+\alpha_{n}^{(5)} v_{n} \\
k_{n+1}=\alpha_{n+1}^{(1)} S_{k} x_{n+1}+\alpha_{n+1}^{(2)} y_{n+1}+\alpha_{n+1}^{(3)} z_{n+1}+\alpha_{n+1}^{(4)} u_{n+1}+\alpha_{n+1}^{(5)} v_{n+1},
\end{gathered}
$$

we compute

$$
\begin{aligned}
\left\|k_{n+1}-k_{n}\right\| \leq & \alpha_{n+1}^{(1)}\left\|S_{k} x_{n+1}-S_{k} x_{n}\right\|+\left|\alpha_{n+1}^{(1)}-\alpha_{n}^{(1)}\right|\left\|S_{k} x_{n}\right\|+\alpha_{n+1}^{(2)}\left\|y_{n+1}-y_{n}\right\| \\
& +\left|\alpha_{n+1}^{(2)}-\alpha_{n}^{(2)}\right|\left\|y_{n}\right\|+\alpha_{n+1}^{(3)}\left\|z_{n+1}-z_{n}\right\|+\left|\alpha_{n+1}^{(3)}-\alpha_{n}^{(3)}\right|\left\|z_{n}\right\| \\
& +\alpha_{n+1}^{(4)}\left\|u_{n+1}-u_{n}\right\|+\left|\alpha_{n+1}^{(4)}-\alpha_{n}^{(4)}\right|\left\|u_{n}\right\|+\alpha_{n+1}^{(5)}\left\|v_{n+1}-v_{n}\right\|+\left|\alpha_{n+1}^{(5)}-\alpha_{n}^{(5)}\right|\left\|v_{n}\right\| \\
\leq & \alpha_{n+1}^{(1)}\left\|x_{n+1}-x_{n}\right\|+\left|\alpha_{n+1}^{(1)}-\alpha_{n}^{(1)}\right|\left\|S_{k} x_{n}\right\|+\alpha_{n+1}^{(2)}\left\|y_{n+1}-y_{n}\right\| \\
& +\left|\alpha_{n+1}^{(2)}-\alpha_{n}^{(2)}\right|\left\|y_{n}\right\|+\alpha_{n+1}^{(3)}\left\|z_{n+1}-z_{n}\right\|+\left|\alpha_{n+1}^{(3)}-\alpha_{n}^{(3)}\right|\left\|z_{n}\right\| \\
& +\alpha_{n+1}^{(4)}\left\|u_{n+1}-u_{n}\right\|+\left|\alpha_{n+1}^{(4)}-\alpha_{n}^{(4)}\right|\left\|u_{n}\right\|+\alpha_{n+1}^{(5)}\left\|v_{n+1}-v_{n}\right\|+\left|\alpha_{n+1}^{(5)}-\alpha_{n}^{(5)}\right|\left\|v_{n}\right\| .
\end{aligned}
$$

Substitution of (3.13), (3.14), (3.15), and (3.16) into (3.18) yields that

$$
\begin{aligned}
\left\|k_{n+1}-k_{n}\right\| \leq & \alpha_{n+1}^{(1)}\left\|x_{n+1}-x_{n}\right\|+\left|\alpha_{n+1}^{(1)}-\alpha_{n}^{(1)}\right|\left\|S_{k} x_{n}\right\| \\
& +\alpha_{n+1}^{(2)}\left\{\left\|x_{n+1}-x_{n}\right\|+\left|\lambda_{n}-\lambda_{n+1}\right|\left\|B x_{n}\right\|\right\}+\left|\alpha_{n+1}^{(2)}-\alpha_{n}^{(2)}\right|\left\|y_{n}\right\| \\
& +\alpha_{n+1}^{(3)}\left\{\left\|x_{n+1}-x_{n}\right\|+\left|\mu_{n}-\mu_{n+1}\right|\left\|C x_{n}\right\|\right\}+\left|\alpha_{n+1}^{(3)}-\alpha_{n}^{(3)}\right|\left\|z_{n}\right\| \\
& +\alpha_{n+1}^{(4)}\left\|x_{n+1}-x_{n}\right\|+\left|\alpha_{n+1}^{(4)}-\alpha_{n}^{(4)}\right|\left\|u_{n}\right\|+\alpha_{n+1}^{(5)}\left\|x_{n+1}-x_{n}\right\|+\left|\alpha_{n+1}^{(5)}-\alpha_{n}^{(5)}\right|\left\|v_{n}\right\| \\
\leq & \left\|x_{n+1}-x_{n}\right\|+M_{1}\left(\left|\alpha_{n+1}^{(1)}-\alpha_{n}^{(1)}\right|+\left|\alpha_{n+1}^{(2)}-\alpha_{n}^{(2)}\right|+\left|\alpha_{n+1}^{(3)}-\alpha_{n}^{(3)}\right|+\left|\alpha_{n+1}^{(4)}-\alpha_{n}^{(4)}\right|\right. \\
& \left.\quad+\left|\alpha_{n+1}^{(5)}-\alpha_{n}^{(5)}\right|+\left|\lambda_{n}-\lambda_{n+1}\right|+\left|\mu_{n}-\mu_{n+1}\right|\right)
\end{aligned}
$$

where $M_{1}$ is an appropriate constant such that

$$
\begin{gathered}
M_{1} \geq \max \left\{\sup _{n \geq 1}\left\{\left\|S_{k} x_{n}\right\|\right\}, \sup _{n \geq 1}\left\{\left\|y_{n}\right\|\right\}, \sup _{n \geq 1}\left\{\left\|z_{n}\right\|\right\}, \sup _{n \geq 1}\left\{\left\|u_{n}\right\|\right\},\right. \\
\left.\sup _{n \geq 1}\left\{\left\|v_{n}\right\|\right\}, \sup _{n \geq 1}\left\{\left\|B x_{n}\right\|\right\}, \sup _{n \geq 1}\left\{\left\|C x_{n}\right\|\right\}\right\} .
\end{gathered}
$$


Fixed Point Theory and Applications

Putting $x_{n+1}=\left(1-\beta_{n}\right) l_{n}+\beta_{n} x_{n}$, for all $n \geq 1$, we have

$$
l_{n}=\frac{x_{n+1}-\beta_{n} x_{n}}{1-\beta_{n}}=\frac{\epsilon_{n} \gamma f\left(x_{n}\right)+\left(\left(1-\beta_{n}\right) I-\epsilon_{n} A\right) k_{n}}{1-\beta_{n}} .
$$

Then, we compute

$$
\begin{aligned}
l_{n+1}-l_{n} & =\frac{\epsilon_{n+1} \gamma f\left(x_{n+1}\right)+\left(\left(1-\beta_{n+1}\right) I-\epsilon_{n+1} A\right) k_{n+1}}{1-\beta_{n+1}}-\frac{\epsilon_{n} \gamma f\left(x_{n}\right)+\left(\left(1-\beta_{n}\right) I-\epsilon_{n} A\right) k_{n}}{1-\beta_{n}} \\
& =\frac{\epsilon_{n+1}}{1-\beta_{n+1}} \gamma f\left(x_{n+1}\right)-\frac{\epsilon_{n}}{1-\beta_{n}} \gamma f\left(x_{n}\right)+k_{n+1}-k_{n}+\frac{\epsilon_{n}}{1-\beta_{n}} A k_{n}-\frac{\epsilon_{n+1}}{1-\beta_{n+1}} A k_{n+1} \\
& =\frac{\epsilon_{n+1}}{1-\beta_{n+1}}\left(\gamma f\left(x_{n+1}\right)-A k_{n+1}\right)+\frac{\epsilon_{n}}{1-\beta_{n}}\left(A k_{n}-\gamma f\left(x_{n}\right)\right)+k_{n+1}-k_{n} .
\end{aligned}
$$

It follows from (3.19) and (3.22) that

$$
\begin{aligned}
\left\|l_{n+1}-l_{n}\right\|-\left\|x_{n+1}-x_{n}\right\| \leq & \frac{\epsilon_{n+1}}{1-\beta_{n+1}}\left(\left\|\gamma f\left(x_{n+1}\right)\right\|+\left\|A k_{n+1}\right\|\right)+\frac{\epsilon_{n}}{1-\beta_{n}}\left(\left\|A k_{n}\right\|+\left\|\gamma f\left(x_{n}\right)\right\|\right) \\
& +\left\|k_{n+1}-k_{n}\right\|-\left\|x_{n+1}-x_{n}\right\| \\
\leq & \frac{\epsilon_{n+1}}{1-\beta_{n+1}}\left(\left\|\gamma f\left(x_{n+1}\right)\right\|+\left\|A k_{n+1}\right\|\right)+\frac{\epsilon_{n}}{1-\beta_{n}}\left(\left\|A k_{n}\right\|+\left\|\gamma f\left(x_{n}\right)\right\|\right) \\
& +M_{1}\left(\left|\alpha_{n+1}^{(1)}-\alpha_{n}^{(1)}\right|+\left|\alpha_{n+1}^{(2)}-\alpha_{n}^{(2)}\right|+\left|\alpha_{n+1}^{(3)}-\alpha_{n}^{(3)}\right|+\left|\alpha_{n+1}^{(4)}-\alpha_{n}^{(4)}\right|\right. \\
& \left.\quad\left|\alpha_{n+1}^{(5)}-\alpha_{n}^{(5)}\right|+\left|\lambda_{n}-\lambda_{n+1}\right|+\left|\mu_{n}-\mu_{n+1}\right|\right) .
\end{aligned}
$$

This together with (C2), (C3), (C4), and (C6) implies that

$$
\limsup _{n \rightarrow \infty}\left(\left\|l_{n+1}-l_{n}\right\|-\left\|x_{n+1}-x_{n}\right\|\right) \leq 0
$$

Hence, by Lemma 2.12, we obtain $\left\|l_{n}-x_{n}\right\| \rightarrow 0$ as $n \rightarrow \infty$. It follows that

$$
\lim _{n \rightarrow \infty}\left\|x_{n+1}-x_{n}\right\|=\lim _{n \rightarrow \infty}\left(1-\beta_{n}\right)\left\|l_{n}-x_{n}\right\|=0 .
$$


Moreover, we also get

$$
\begin{aligned}
\lim _{n \rightarrow \infty}\left\|u_{n+1}-u_{n}\right\| & =\lim _{n \rightarrow \infty}\left\|v_{n+1}-v_{n}\right\|=\lim _{n \rightarrow \infty}\left\|z_{n+1}-z_{n}\right\|=\lim _{n \rightarrow \infty}\left\|y_{n+1}-y_{n}\right\| \\
& =\lim _{n \rightarrow \infty}\left\|k_{n+1}-k_{n}\right\|=0 .
\end{aligned}
$$

Observe that

$$
x_{n+1}-x_{n}=\epsilon_{n}\left(\gamma f\left(x_{n}\right)-A x_{n}\right)+\left(1-\beta_{n}-\epsilon_{n} \bar{\gamma}\right)\left(k_{n}-x_{n}\right) .
$$

By conditions (C2), (C3), and (3.25), we have

$$
\lim _{n \rightarrow \infty}\left\|k_{n}-x_{n}\right\|=0 .
$$

Step 3. We claim that the following statements hold:

(s1) $\lim _{n \rightarrow \infty}\left\|x_{n}-u_{n}\right\|=0$;

(s2) $\lim _{n \rightarrow \infty}\left\|x_{n}-y_{n}\right\|=0$;

(s3) $\lim _{n \rightarrow \infty}\left\|x_{n}-z_{n}\right\|=0$;

(s4) $\lim _{n \rightarrow \infty}\left\|x_{n}-v_{n}\right\|=0$.

For $p \in \Theta$, we compute

$$
\begin{aligned}
\left\|z_{n}-p\right\|^{2} & =\left\|P_{E}\left(x_{n}-\mu_{n} C x_{n}\right)-P_{E}\left(p-\mu_{n} C p\right)\right\|^{2} \\
& \leq\left\|\left(x_{n}-\mu_{n} C x_{n}\right)-\left(p-\mu_{n} C p\right)\right\|^{2} \\
& =\left\|\left(x_{n}-p\right)-\mu_{n}\left(C x_{n}-C p\right)\right\|^{2} \\
& \leq\left\|x_{n}-p\right\|^{2}-2 \mu_{n}\left\langle x_{n}-p, C x_{n}-C p\right\rangle+\mu_{n}^{2}\left\|C x_{n}-C p\right\|^{2} \\
& \leq\left\|x_{n}-p\right\|^{2}+\mu_{n}\left(\mu_{n}-2 \xi\right)\left\|C x_{n}-C p\right\|^{2} \\
& =\left\|x_{n}-p\right\|^{2}-\mu_{n}\left(2 \xi-\mu_{n}\right)\left\|C x_{n}-C p\right\|^{2} .
\end{aligned}
$$

By the same way, we can get

$$
\left\|y_{n}-p\right\|^{2} \leq\left\|x_{n}-p\right\|^{2}-\lambda_{n}\left(2 \beta-\lambda_{n}\right)\left\|B x_{n}-B p\right\|^{2} .
$$


We note that

$$
\begin{aligned}
\left\|u_{n}-p\right\|^{2} & =\left\|T_{r}^{\Phi_{1}}\left(I-r \Psi_{1}\right) x_{n}-T_{r}^{\Phi_{1}}\left(I-r \Psi_{1}\right) p\right\|^{2} \\
& \leq\left\|\left(I-r \Psi_{1}\right) x_{n}-\left(I-r \Psi_{1}\right) p\right\|^{2} \\
& =\left\|\left(x_{n}-p\right)-r\left(\Psi_{1} x_{n}-\Psi_{1}\right) p\right\|^{2} \\
& =\left\|x_{n}-p\right\|^{2}-2 r\left\langle x_{n}-p, \Psi_{1} x_{n}-\Psi_{1} p\right\rangle+r^{2}\left\|\Psi_{1} x_{n}-\Psi_{1} p\right\|^{2} \\
& \leq\left\|x_{n}-p\right\|^{2}-2 r \rho\left\|\Psi_{1} x_{n}-\Psi_{1} p\right\|+r^{2}\left\|\Psi_{1} x_{n}-\Psi_{1} p\right\|^{2} \\
& =\left\|x_{n}-p\right\|^{2}+r(r-2 \rho)\left\|\Psi_{1} x_{n}-\Psi_{1} p\right\|^{2} \\
& =\left\|x_{n}-p\right\|^{2}-r(2 \rho-r)\left\|\Psi_{1} x_{n}-\Psi_{1} p\right\|^{2} .
\end{aligned}
$$

Similarly, we have

$$
\left\|v_{n}-p\right\|^{2} \leq\left\|x_{n}-p\right\|^{2}-s(2 \omega-s)\left\|\Psi_{2} x_{n}-\Psi_{2} p\right\|^{2} .
$$

Observe that

$$
\begin{aligned}
\left\|k_{n}-p\right\|^{2} & \leq \alpha_{n}^{(1)}\left\|S_{k} x_{n}-p\right\|^{2}+\alpha_{n}^{(2)}\left\|y_{n}-p\right\|^{2}+\alpha_{n}^{(3)}\left\|z_{n}-p\right\|^{2}+\alpha_{n}^{(4)}\left\|u_{n}-p\right\|^{2}+\alpha_{n}^{(5)}\left\|v_{n}-p\right\|^{2} \\
& \leq \alpha_{n}^{(1)}\left\|x_{n}-p\right\|^{2}+\alpha_{n}^{(2)}\left\|y_{n}-p\right\|^{2}+\alpha_{n}^{(3)}\left\|z_{n}-p\right\|^{2}+\alpha_{n}^{(4)}\left\|u_{n}-p\right\|^{2}+\alpha_{n}^{(5)}\left\|v_{n}-p\right\|^{2}
\end{aligned}
$$

Substituting (3.29), (3.30), (3.31), and (3.32) into (3.33), we obtain

$$
\begin{aligned}
\left\|k_{n}-p\right\|^{2} \leq & \alpha_{n}^{(1)}\left\|x_{n}-p\right\|^{2}+\alpha_{n}^{(2)}\left\{\left\|x_{n}-p\right\|^{2}-\lambda_{n}\left(2 \beta-\lambda_{n}\right)\left\|B x_{n}-B p\right\|^{2}\right\} \\
& +\alpha_{n}^{(3)}\left\{\left\|x_{n}-p\right\|^{2}-\mu_{n}\left(2 \xi-\mu_{n}\right)\left\|C x_{n}-C p\right\|^{2}\right\} \\
& +\alpha_{n}^{(4)}\left\{\left\|x_{n}-p\right\|^{2}-r(2 \rho-r)\left\|\Psi_{1} x_{n}-\Psi_{1} p\right\|^{2}\right\} \\
& +\alpha_{n}^{(5)}\left\{\left\|x_{n}-p\right\|^{2}-s(2 \omega-s)\left\|\Psi_{2} x_{n}-\Psi_{2} p\right\|^{2}\right\} \\
= & \left\|x_{n}-p\right\|^{2}-\alpha_{n}^{(2)} \lambda_{n}\left(2 \beta-\lambda_{n}\right)\left\|B x_{n}-B p\right\|^{2}-\alpha_{n}^{(3)} \mu_{n}\left(2 \xi-\mu_{n}\right)\left\|C x_{n}-C p\right\|^{2} \\
& -\alpha_{n}^{(4)} r(2 \rho-r)\left\|\Psi_{1} x_{n}-\Psi_{1} p\right\|^{2}-\alpha_{n}^{(5)} s(2 \omega-s)\left\|\Psi_{2} x_{n}-\Psi_{2} p\right\|^{2} .
\end{aligned}
$$


It follows from (3.2) and (3.34) that

$$
\begin{aligned}
&\left\|x_{n+1}-p\right\|^{2} \\
&=\left\|\epsilon_{n} \gamma f\left(x_{n}\right)+\beta_{n} x_{n}+\left(\left(1-\beta_{n}\right) I-\epsilon_{n} A\right) k_{n}-p\right\|^{2} \\
& \leq \epsilon_{n}\left\|\gamma f\left(x_{n}\right)-A p\right\|^{2}+\beta_{n}\left\|x_{n}-p\right\|^{2}+\left(1-\beta_{n}-\epsilon_{n} \bar{\gamma}\right)\left\|k_{n}-p\right\|^{2} \\
& \leq \epsilon_{n}\left\|\gamma f\left(x_{n}\right)-A p\right\|^{2}+\beta_{n}\left\|x_{n}-p\right\|^{2}+\left(1-\beta_{n}-\epsilon_{n} \bar{\gamma}\right) \\
& \quad \times\left\{\left\|x_{n}-p\right\|^{2}-\alpha_{n}^{(2)} \lambda_{n}\left(2 \beta-\lambda_{n}\right)\left\|B x_{n}-B p\right\|^{2}-\alpha_{n}^{(3)} \mu_{n}\left(2 \xi-\mu_{n}\right)\left\|C x_{n}-C p\right\|^{2}\right. \\
&\left.\quad-\alpha_{n}^{(4)} r(2 \rho-r)\left\|\Psi_{1} x_{n}-\Psi_{1} p\right\|^{2}-\alpha_{n}^{(5)} s(2 \omega-s)\left\|\Psi_{2} x_{n}-\Psi_{2} p\right\|^{2}\right\} \\
&=\epsilon_{n}\left\|\gamma f\left(x_{n}\right)-A p\right\|^{2}+\left(1-\epsilon_{n} \bar{\gamma}\right)\left\|x_{n}-p\right\|^{2}-\left(1-\beta_{n}-\epsilon_{n} \bar{\gamma}\right) \alpha_{n}^{(2)} \lambda_{n}\left(2 \beta-\lambda_{n}\right)\left\|B x_{n}-B p\right\|^{2} \\
& \quad-\left(1-\beta_{n}-\epsilon_{n} \bar{\gamma}\right) \alpha_{n}^{(3)} \mu_{n}\left(2 \xi-\mu_{n}\right)\left\|C x_{n}-C p\right\|^{2} \\
& \quad-\left(1-\beta_{n}-\epsilon_{n} \bar{\gamma}\right) \alpha_{n}^{(4)} r(2 \rho-r)\left\|\Psi_{1} x_{n}-\Psi_{1} p\right\|^{2} \\
& \quad-\left(1-\beta_{n}-\epsilon_{n} \bar{\gamma}\right) \alpha_{n}^{(5)} s(2 \omega-s)\left\|\Psi_{2} x_{n}-\Psi_{2} p\right\|^{2} \\
& \leq \epsilon_{n}\left\|\gamma f\left(x_{n}\right)-A p\right\|^{2}+\left\|x_{n}-p\right\|^{2}-\left(1-\beta_{n}-\epsilon_{n} \bar{\gamma}\right) \alpha_{n}^{(2)} \lambda_{n}\left(2 \beta-\lambda_{n}\right)\left\|B x_{n}-B p\right\|^{2} \\
& \quad-\left(1-\beta_{n}-\epsilon_{n} \bar{\gamma}\right) \alpha_{n}^{(3)} \mu_{n}\left(2 \xi-\mu_{n}\right)\left\|C x_{n}-C p\right\|^{2} \\
& \quad-\left(1-\beta_{n}-\epsilon_{n} \bar{\gamma}\right) \alpha_{n}^{(4)} r(2 \rho-r)\left\|\Psi_{1} x_{n}-\Psi_{1} p\right\|^{2} \\
&-\left(1-\beta_{n}-\epsilon_{n} \bar{\gamma}\right) \alpha_{n}^{(5)} s(2 \omega-s)\left\|\Psi_{2} x_{n}-\Psi_{2} p\right\|^{2} \\
& \leq \epsilon_{n}\left\|\gamma f\left(x_{n}\right)-A p\right\|^{2}+\left\|x_{n}-p\right\|^{2}-\left(1-\beta_{n}-\epsilon_{n} \bar{\gamma}\right) \alpha_{n}^{(3)} \mu_{n}\left(2 \xi-\mu_{n}\right)\left\|C x_{n}-C p\right\|^{2} .
\end{aligned}
$$

It follows from (C5) that

$$
\begin{aligned}
(1- & \left.\beta_{n}-\epsilon_{n} \bar{\gamma}\right) \alpha_{n}^{(3)} \mu_{n}\left(2 \xi-\mu_{n}\right)\left\|C x_{n}-C p\right\|^{2} \\
& \leq \epsilon_{n}\left\|\gamma f\left(x_{n}\right)-A p\right\|^{2}+\left\|x_{n}-p\right\|^{2}-\left\|x_{n+1}-p\right\|^{2} \\
& =\epsilon_{n}\left\|\gamma f\left(x_{n}\right)-A p\right\|^{2}+\left(\left\|x_{n}-p\right\|-\left\|x_{n+1}-p\right\|\right)\left(\left\|x_{n}-p\right\|+\left\|x_{n+1}-p\right\|\right) \\
& \leq \epsilon_{n}\left\|r f\left(x_{n}\right)-A p\right\|^{2}+\left\|x_{n+1}-x_{n}\right\|\left(\left\|x_{n}-p\right\|+\left\|x_{n+1}-p\right\|\right) .
\end{aligned}
$$

From (C2), (C6), and (3.25), we have

$$
\lim _{n \rightarrow \infty}\left\|C x_{n}-C p\right\|=0 .
$$


Since $s \in(0,2 \omega)$, we also have

$$
\begin{aligned}
& \left(1-\beta_{n}-\epsilon_{n} \bar{\gamma}\right) \alpha_{n}^{(5)} s(2 \omega-s)\left\|\Psi_{2} x_{n}-\Psi_{2} p\right\|^{2} \\
& \quad \leq \epsilon_{n}\left\|\gamma f\left(x_{n}\right)-A p\right\|^{2}+\left\|x_{n}-p\right\|^{2}-\left\|x_{n+1}-p\right\|^{2} \\
& \quad \leq \epsilon_{n}\left\|\gamma f\left(x_{n}\right)-A p\right\|^{2}+\left\|x_{n+1}-x_{n}\right\|\left(\left\|x_{n}-p\right\|+\left\|x_{n+1}-p\right\|\right) .
\end{aligned}
$$

From (C2), (C6), and (3.25), we obtain

$$
\lim _{n \rightarrow \infty}\left\|\Psi_{2} x_{n}-\Psi_{2} p\right\|=0
$$

Similarly, from (3.37) and (3.39), we can prove that

$$
\lim _{n \rightarrow \infty}\left\|B x_{n}-B p\right\|=\lim _{n \rightarrow \infty}\left\|\Psi_{1} x_{n}-\Psi_{1} p\right\|=0
$$

On the other hand, let $p \in \Theta$ for each $n \geq 1$; we get $p=T_{r}^{\Phi_{1}}\left(I-r \Psi_{1}\right) p$. By Lemma 2.10(iii), that is, $T_{r}^{\Phi_{1}}$ is firmly nonexpansive, we obtain

$$
\begin{aligned}
\| u_{n} & -p \|^{2} \\
& =\left\|T_{r}^{\Phi_{1}}\left(I-r \Psi_{1}\right) x_{n}-T_{r}^{\Phi_{1}}\left(I-r \Psi_{1}\right) p\right\|^{2} \\
& \leq\left\langle\left(I-r \Psi_{1}\right) x_{n}-\left(I-r \Psi_{1}\right) p, u_{n}-p\right\rangle \\
& =\frac{1}{2}\left\{\left\|\left(I-r \Psi_{1}\right) x_{n}-\left(I-r \Psi_{1}\right) p\right\|^{2}+\left\|u_{n}-p\right\|^{2}-\left\|\left(I-r \Psi_{1}\right) x_{n}-\left(I-r \Psi_{1}\right) p-\left(u_{n}-p\right)\right\|^{2}\right\} \\
& \leq \frac{1}{2}\left\{\left\|x_{n}-p\right\|^{2}+\left\|u_{n}-p\right\|^{2}-\left\|\left(x_{n}-u_{n}\right)-r\left(\Psi_{1} x_{n}-\Psi_{1} p\right)\right\|^{2}\right\} \\
& \leq \frac{1}{2}\left\{\left\|x_{n}-p\right\|^{2}+\left\|u_{n}-p\right\|^{2}-\left\|x_{n}-u_{n}\right\|^{2}+2 r\left\|x_{n}-u_{n}\right\|\left\|\Psi_{1} x_{n}-\Psi_{1} p\right\|-r^{2}\left\|\Psi_{1} x_{n}-\Psi_{1} p\right\|^{2}\right\} .
\end{aligned}
$$

So, we obtain

$$
\left\|u_{n}-p\right\|^{2} \leq\left\|x_{n}-p\right\|^{2}-\left\|x_{n}-u_{n}\right\|^{2}+2 r\left\|x_{n}-u_{n}\right\|\left\|\Psi_{1} x_{n}-\Psi_{1} p\right\| .
$$


Observe that

$$
\begin{aligned}
\left\|y_{n}-p\right\|^{2}= & \left\|P_{E}\left(x_{n}-\lambda_{n} B x_{n}\right)-P_{E}\left(p-\lambda_{n} B p\right)\right\|^{2} \\
\leq & \left\langle\left(I-\lambda_{n} B\right) x_{n}-\left(I-\lambda_{n} B\right) p, y_{n}-p\right\rangle \\
= & \frac{1}{2}\left\{\left\|\left(I-\lambda_{n} B\right) x_{n}-\left(I-\lambda_{n} B\right) p\right\|^{2}+\left\|y_{n}-p\right\|^{2}\right. \\
& \left.\quad-\left\|\left(I-\lambda_{n} B\right) x_{n}-\left(I-\lambda_{n} B\right) p-\left(y_{n}-p\right)\right\|^{2}\right\} \\
\leq & \frac{1}{2}\left\{\left\|x_{n}-p\right\|^{2}+\left\|y_{n}-p\right\|^{2}-\left\|\left(x_{n}-y_{n}\right)-\lambda_{n}\left(B x_{n}-B p\right)\right\|^{2}\right\} \\
\leq & \frac{1}{2}\left\{\left\|x_{n}-p\right\|^{2}+\left\|y_{n}-p\right\|^{2}-\left\|x_{n}-y_{n}\right\|^{2}-\lambda_{n}^{2}\left\|B x_{n}-B p\right\|^{2}\right. \\
& \left.\quad+2 \lambda_{n}\left\langle x_{n}-y_{n}, B x_{n}-B p\right\rangle\right\},
\end{aligned}
$$

and hence

$$
\left\|y_{n}-p\right\|^{2} \leq\left\|x_{n}-p\right\|^{2}-\left\|x_{n}-y_{n}\right\|^{2}+2 \lambda_{n}\left\|x_{n}-y_{n}\right\|\left\|B x_{n}-B p\right\| .
$$

By using the same argument in (3.42) and (3.44), we can prove that

$$
\begin{aligned}
& \left\|v_{n}-p\right\|^{2} \leq\left\|x_{n}-p\right\|^{2}-\left\|x_{n}-v_{n}\right\|^{2}+2 s\left\|x_{n}-v_{n}\right\|\left\|\Psi_{2} x_{n}-\Psi_{2} p\right\|, \\
& \left\|z_{n}-p\right\|^{2} \leq\left\|x_{n}-p\right\|^{2}-\left\|x_{n}-z_{n}\right\|^{2}+2 \mu_{n}\left\|x_{n}-z_{n}\right\|\left\|C x_{n}-C p\right\| .
\end{aligned}
$$

Substituting (3.42), (3.44), and (3.45) into (3.33), we obtain

$$
\begin{aligned}
\left\|k_{n}-p\right\|^{2} \leq & \alpha_{n}^{(1)}\left\|x_{n}-p\right\|^{2}+\alpha_{n}^{(2)}\left\|y_{n}-p\right\|^{2}+\alpha_{n}^{(3)}\left\|z_{n}-p\right\|^{2}+\alpha_{n}^{(4)}\left\|u_{n}-p\right\|^{2}+\alpha_{n}^{(5)}\left\|v_{n}-p\right\|^{2} \\
\leq & \alpha_{n}^{(1)}\left\|x_{n}-p\right\|^{2}+\alpha_{n}^{(2)}\left\{\left\|x_{n}-p\right\|^{2}-\left\|x_{n}-y_{n}\right\|^{2}+2 \lambda_{n}\left\|x_{n}-y_{n}\right\|\left\|B x_{n}-B p\right\|\right\} \\
& +\alpha_{n}^{(3)}\left\{\left\|x_{n}-p\right\|^{2}-\left\|x_{n}-z_{n}\right\|^{2}+2 \mu_{n}\left\|x_{n}-z_{n}\right\|\left\|C x_{n}-C p\right\|\right\} \\
& +\alpha_{n}^{(4)}\left\{\left\|x_{n}-p\right\|^{2}-\left\|x_{n}-u_{n}\right\|^{2}+2 r\left\|x_{n}-u_{n}\right\|\left\|\Psi_{1} x_{n}-\Psi_{1} p\right\|\right\} \\
& +\alpha_{n}^{(5)}\left\{\left\|x_{n}-p\right\|^{2}-\left\|x_{n}-v_{n}\right\|^{2}+2 s\left\|x_{n}-v_{n}\right\|\left\|\Psi_{2} x_{n}-\Psi_{2} p\right\|\right\} \\
= & \left\|x_{n}-p\right\|^{2}-\alpha_{n}^{(2)}\left\|x_{n}-y_{n}\right\|^{2}+2 \lambda_{n} \alpha_{n}^{(2)}\left\|x_{n}-y_{n}\right\|\left\|B x_{n}-B p\right\| \\
& -\alpha_{n}^{(3)}\left\|x_{n}-z_{n}\right\|^{2}+2 \mu_{n} \alpha_{n}^{(3)}\left\|x_{n}-z_{n}\right\|\left\|C x_{n}-C p\right\| \\
& -\alpha_{n}^{(4)}\left\|x_{n}-u_{n}\right\|^{2}+2 r \alpha_{n}^{(4)}\left\|x_{n}-u_{n}\right\|\left\|\Psi_{1} x_{n}-\Psi_{1} p\right\| \\
& -\alpha_{n}^{(5)}\left\|x_{n}-v_{n}\right\|^{2}+2 s \alpha_{n}^{(5)}\left\|x_{n}-v_{n}\right\|\left\|\Psi_{2} x_{n}-\Psi_{2} p\right\| .
\end{aligned}
$$


From Lemma 2.4, (3.2), and (3.46), we obtain

$$
\begin{aligned}
\left\|x_{n+1}-p\right\|^{2}= & \left\|\epsilon_{n}\left(\gamma f\left(x_{n}\right)-A p\right)+\beta_{n}\left(x_{n}-p\right)+\left(\left(1-\beta_{n}\right) I-\epsilon_{n} A\right)\left(k_{n}-p\right)\right\|^{2} \\
\leq & \epsilon_{n}\left\|\gamma f\left(x_{n}\right)-A p\right\|^{2}+\beta_{n}\left\|x_{n}-p\right\|^{2}+\left(1-\beta_{n}-\epsilon_{n} \bar{\gamma}\right)\left\|k_{n}-p\right\|^{2} \\
\leq & \epsilon_{n}\left\|\gamma f\left(x_{n}\right)-A p\right\|^{2}+\beta_{n}\left\|x_{n}-p\right\|^{2} \\
& +\left(1-\beta_{n}-\epsilon_{n} \bar{\gamma}\right)\left\{\left\|x_{n}-p\right\|^{2}-\alpha_{n}^{(2)}\left\|x_{n}-y_{n}\right\|^{2}+2 \lambda_{n} \alpha_{n}^{(2)}\left\|x_{n}-y_{n}\right\|\left\|B x_{n}-B p\right\|\right. \\
& \quad-\alpha_{n}^{(3)}\left\|x_{n}-z_{n}\right\|^{2}+2 \mu_{n} \alpha_{n}^{(3)}\left\|x_{n}-z_{n}\right\|\left\|C x_{n}-C p\right\| \\
& \quad-\alpha_{n}^{(4)}\left\|x_{n}-u_{n}\right\|^{2}+2 r \alpha_{n}^{(4)}\left\|x_{n}-u_{n}\right\|\left\|\Psi_{1} x_{n}-\Psi_{1} p\right\| \\
& \left.\quad \alpha_{n}^{(5)}\left\|x_{n}-v_{n}\right\|^{2}+2 s \alpha_{n}^{(5)}\left\|x_{n}-v_{n}\right\|\left\|\Psi_{2} x_{n}-\Psi_{2} p\right\|\right\} \\
& -\left(1-\beta_{n}-\epsilon_{n} \bar{\gamma}\right) \alpha_{n}^{(2)}\left\|x_{n}-y_{n}\right\|^{2}+2\left(1-\beta_{n}-\epsilon_{n} \bar{\gamma}\right) \lambda_{n} \alpha_{n}^{(2)}\left\|x_{n}-y_{n}\right\|\left\|B x_{n}-B p\right\| \\
& -\left(1-\beta_{n}-\epsilon_{n} \bar{\gamma}\right) \alpha_{n}^{(3)}\left\|x_{n}-z_{n}\right\|^{2}+2\left(1-\beta_{n}-\epsilon_{n} \bar{\gamma}\right) \mu_{n} \alpha_{n}^{(3)}\left\|x_{n}-z_{n}\right\|\left\|C x_{n}-C p\right\| \\
& -\left(1-\beta_{n}-\epsilon_{n} \bar{\gamma}\right) \alpha_{n}^{(4)}\left\|x_{n}-u_{n}\right\|^{2}+2 r\left(1-\beta_{n}-\epsilon_{n} \bar{\gamma}\right) \alpha_{n}^{(4)}\left\|x_{n}-u_{n}\right\|\left\|\Psi_{1} x_{n}-\Psi_{1} p\right\| \\
& -\left(1-\beta_{n}-\epsilon_{n} \bar{\gamma}\right) \alpha_{n}^{(5)}\left\|x_{n}-v_{n}\right\|^{2}+2 s\left(1-\beta_{n}-\epsilon_{n} \bar{\gamma}\right) \alpha_{n}^{(5)}\left\|x_{n}-v_{n}\right\|\left\|\Psi_{2} x_{n}-\Psi_{2} p\right\| \\
\leq & \epsilon_{n}\left\|\gamma f\left(x_{n}\right)-A p\right\|^{2}+\left\|x_{n}-p\right\|^{2}-\left(1-\beta_{n}-\epsilon_{n} \bar{\gamma}\right) \alpha_{n}^{(2)}\left\|x_{n}-y_{n}\right\|^{2} \\
& +2\left(1-\beta_{n}-\epsilon_{n} \bar{\gamma}\right) \lambda_{n} \alpha_{n}^{(2)}\left\|x_{n}-y_{n}\right\|\left\|B x_{n}-B p\right\|-\left(1-\beta_{n}-\epsilon_{n} \bar{\gamma}\right) \alpha_{n}^{(3)}\left\|x_{n}-z_{n}\right\|^{2} \\
& +2\left(1-\beta_{n}-\epsilon_{n} \bar{\gamma}\right) \mu_{n} \alpha_{n}^{(3)}\left\|x_{n}-z_{n}\right\|\left\|C x_{n}-C p\right\|-\left(1-\beta_{n}-\epsilon_{n} \bar{\gamma}\right) \alpha_{n}^{(4)}\left\|x_{n}-u_{n}\right\|^{2} \\
& +2 r\left(1-\beta_{n}-\epsilon_{n} \bar{\gamma}\right) \alpha_{n}^{(4)}\left\|x_{n}-u_{n}\right\|\left\|\Psi_{1} x_{n}-\Psi_{1} p\right\|-\left(1-\beta_{n}-\epsilon_{n} \bar{\gamma}\right) \alpha_{n}^{(5)}\left\|x_{n}-v_{n}\right\|^{2} \\
& +2 s\left(1-\beta_{n}-\epsilon_{n} \bar{\gamma}\right) \alpha_{n}^{(5)}\left\|x_{n}-v_{n}\right\|\left\|\Psi_{2} x_{n}-\Psi_{2} p\right\| .
\end{aligned}
$$

It follows that

$$
\begin{aligned}
\left(1-\beta_{n}-\epsilon_{n} \bar{\gamma}\right) \alpha_{n}^{(4)}\left\|x_{n}-u_{n}\right\|^{2} \leq & \epsilon_{n}\left\|\gamma f\left(x_{n}\right)-A p\right\|^{2}+\left\|x_{n+1}-x_{n}\right\|\left(\left\|x_{n}-p\right\|+\left\|x_{n+1}-p\right\|\right) \\
& +2\left(1-\beta_{n}-\epsilon_{n} \bar{\gamma}\right) \lambda_{n} \alpha_{n}^{(2)}\left\|x_{n}-y_{n}\right\|\left\|B x_{n}-B p\right\| \\
& +2\left(1-\beta_{n}-\epsilon_{n} \bar{\gamma}\right) \mu_{n} \alpha_{n}^{(3)}\left\|x_{n}-z_{n}\right\|\left\|C x_{n}-C p\right\| \\
+ & 2 r\left(1-\beta_{n}-\epsilon_{n} \bar{\gamma}\right) \alpha_{n}^{(4)}\left\|x_{n}-u_{n}\right\|\left\|\Psi_{1} x_{n}-\Psi_{1} p\right\| \\
+ & 2 s\left(1-\beta_{n}-\epsilon_{n} \bar{\gamma}\right) \alpha_{n}^{(5)}\left\|x_{n}-v_{n}\right\|\left\|\Psi_{2} x_{n}-\Psi_{2} p\right\| .
\end{aligned}
$$


From (C2), (C6), (3.37), (3.39), (3.40), and $\left\|x_{n+1}-x_{n}\right\| \rightarrow 0$ as $n \rightarrow \infty$, we also have

$$
\lim _{n \rightarrow \infty}\left\|x_{n}-u_{n}\right\|=0
$$

From (3.47) and by using the same argument above, we can prove that

$$
\lim _{n \rightarrow \infty}\left\|x_{n}-y_{n}\right\|=\lim _{n \rightarrow \infty}\left\|x_{n}-z_{n}\right\|=\lim _{n \rightarrow \infty}\left\|x_{n}-v_{n}\right\|=0
$$

Applying (3.28), (3.49), and (3.50), we obtain

$$
\lim _{n \rightarrow \infty}\left\|k_{n}-u_{n}\right\|=\lim _{n \rightarrow \infty}\left\|k_{n}-y_{n}\right\|=\lim _{n \rightarrow \infty}\left\|k_{n}-z_{n}\right\|=\lim _{n \rightarrow \infty}\left\|k_{n}-v_{n}\right\|=0
$$

Step 4. We claim that $\lim \sup _{n \rightarrow \infty}\left\langle(A-\gamma f) q, q-x_{n}\right\rangle \leq 0$, where $q=P_{\Theta}(I-A+\gamma f)(q)$ is the unique solution of the variational inequality $\langle(A-\gamma f) q, x-q\rangle \geq 0$, for all $x \in \Theta$.

To show the above inequality, we choose a subsequence $\left\{x_{n_{i}}\right\}$ of $\left\{x_{n}\right\}$ such that

$$
\limsup _{n \rightarrow \infty}\left\langle(A-\gamma f) q, q-x_{n}\right\rangle=\lim _{i \rightarrow \infty}\left\langle(A-\gamma f) q, q-x_{n_{i}}\right\rangle
$$

Since $\left\{x_{n_{i}}\right\}$ is bounded, there exists a subsequence $\left\{x_{n_{i j}}\right\}$ of $\left\{x_{n_{i}}\right\}$ which converges weakly to $z \in E$. Without loss of generality, we can assume that $x_{n_{i}} \rightarrow z$. We claim that $z \in \Theta$.

That is, we will prove that

$$
z \in F(S) \cap \operatorname{VI}(E, C) \cap \operatorname{VI}(E, B) \cap \operatorname{GMEP}\left(\Phi_{1}, \varphi, \Psi_{1}\right) \cap \operatorname{GMEP}\left(\Phi_{2}, \varphi, \Psi_{2}\right)
$$

Assume also that $\lambda_{n} \rightarrow \lambda \in[d, 2 \beta]$ and $\mu_{n} \rightarrow \mu \in[e, 2 \xi]$.

Define a mapping $Q: E \rightarrow E$ by

$$
\begin{aligned}
\mathcal{Q} x= & \alpha^{(1)} S_{k} x+\alpha^{(2)} P_{E}(1-\lambda B) x+\alpha^{(3)} P_{E}(1-\mu C) x+\alpha^{(4)} T_{r}^{\Phi_{1}}\left(I-r \Psi_{1}\right) x \\
& +\alpha^{(5)} T_{s}^{\Phi_{2}}\left(I-r \Psi_{2}\right) x, \quad \forall x \in E,
\end{aligned}
$$

where $\lim _{n \rightarrow \infty} \alpha_{n}^{(i)}=\alpha^{(i)} \in(0,1)$, where $i=1,2,3,4,5$. Since $\sum_{i=1}^{5} \alpha_{n}^{(i)}=1$ and by Lemma 2.9, we have that $Q$ is nonexpansive and

$$
\begin{aligned}
F(\mathcal{Q}) & =F\left(S_{k}\right) \cap F\left(P_{E}(1-\lambda B)\right) \cap F\left(P_{E}(1-\mu C)\right) \cap F\left(T_{r}^{\Phi_{1}}\left(I-r \Psi_{1}\right)\right) \cap F\left(T_{s}^{\Phi_{2}}\left(I-r \Psi_{2}\right)\right) \\
& =F(S) \cap \operatorname{VI}(E, C) \cap \operatorname{VI}(E, B) \cap \operatorname{GMEP}\left(\Phi_{1}, \varphi, \Psi_{1}\right) \cap \operatorname{GMEP}\left(\Phi_{2}, \varphi, \Psi_{2}\right) .
\end{aligned}
$$


Notice that

$$
\begin{aligned}
& \left\|Q x_{n}-x_{n}\right\| \\
& \leq\left\|Q x_{n}-k_{n}\right\|+\left\|k_{n}-x_{n}\right\| \\
& =\|\left[\alpha^{(1)} S_{k} x_{n}+\alpha^{(2)} P_{E}(1-\lambda B) x_{n}+\alpha^{(3)} P_{E}(1-\mu C) x_{n}+\alpha^{(4)} T_{r}^{\Phi_{1}}\left(I-r \Psi_{1}\right) x_{n}\right. \\
& \left.\quad+\alpha^{(5)} T_{s}^{\Phi_{2}}\left(I-r \Psi_{2}\right) x_{n}\right]-\left[\alpha_{n}^{(1)} S_{k} x_{n}+\alpha_{n}^{(2)} P_{E}\left(1-\lambda_{n} B\right) x_{n}+\alpha_{n}^{(3)} P_{E}\left(1-\mu_{n} C\right) x_{n}\right. \\
& \left.\quad+\alpha_{n}^{(4)} T_{r}^{\Phi_{1}}\left(I-r \Psi_{1}\right) x_{n}+\alpha_{n}^{(5)} T_{s}^{\Phi_{2}}\left(I-r \Psi_{2}\right) x_{n}\right]\|+\| k_{n}-x_{n} \| \\
& \leq\left|\alpha^{(1)}-\alpha_{n}^{(1)}\right|\left\|S_{k} x_{n}\right\|+\alpha^{(2)}\left\|P_{E}(I-\lambda B) x_{n}-P_{E}\left(I-\lambda_{n} B\right) x_{n}\right\|+\left|\alpha^{(2)}-\alpha_{n}^{(2)}\right|\left\|P_{E}\left(I-\lambda_{n} B\right) x_{n}\right\| \\
& \quad+\alpha^{(3)}\left\|P_{E}(I-\mu C) x_{n}-P_{E}\left(I-\mu_{n} C\right) x_{n}\right\|+\left|\alpha^{(3)}-\alpha_{n}^{(3)}\right|\left\|P_{E}\left(I-\mu_{n} C\right) x_{n}\right\| \\
& \quad+\left|\alpha^{(4)}-\alpha_{n}^{(4)}\right|\left\|T_{r}^{\Phi_{1}}\left(I-r \Psi_{1}\right) x_{n}\right\|+\left|\alpha^{(5)}-\alpha_{n}^{(5)}\right|\left\|T_{s}^{\Phi_{2}}\left(I-r \Psi_{2}\right) x_{n}\right\|+\left\|k_{n}-x_{n}\right\| \\
& \leq\left|\alpha^{(1)}-\alpha_{n}^{(1)}\right|\left\|S_{k} x_{n}\right\|+\alpha^{(2)}\left|\lambda_{n}-\lambda\right|\left\|B x_{n}\right\|+\left|\alpha^{(2)}-\alpha_{n}^{(2)}\right|\left\|P_{E}\left(I-\lambda_{n} B\right) x_{n}\right\| \\
& \quad+\alpha^{(3)}\left|\mu_{n}-\mu\right|\left\|C x_{n}\right\|+\left|\alpha^{(3)}-\alpha_{n}^{(3)}\right|\left\|P_{E}\left(I-\mu_{n} C\right) x_{n}\right\| \\
& \quad+\left|\alpha^{(4)}-\alpha_{n}^{(4)}\right||| T_{r}^{\Phi_{1}}\left(I-r \Psi_{1}\right) x_{n}\left\|+\left|\alpha^{(5)}-\alpha_{n}^{(5)}\right|\right\| T_{s}^{\Phi_{2}}\left(I-r \Psi_{2}\right) x_{n}\|+\| k_{n}-x_{n} \| \\
& \leq \\
& \quad K_{1}\left(\sum_{i=1}^{5}\left|\alpha^{(i)}-\alpha_{n}^{(i)}\right|+\left|\lambda_{n}-\lambda\right|+\left|\mu_{n}-\mu\right|\right)+\left\|k_{n}-x_{n}\right\|,
\end{aligned}
$$

where $K_{1}$ is an appropriate constant such that

$$
\begin{gathered}
K_{1} \geq \max \left\{\sup _{n \geq 1}\left\{\left\|T_{r}^{\Phi_{1}}\left(I-r \Psi_{1}\right) x_{n}\right\|\right\}, \sup _{n \geq 1}\left\{\left\|T_{s}^{\Phi_{2}}\left(I-r \Psi_{2}\right) x_{n}\right\|\right\}, \sup _{n \geq 1}\left\{\left\|P_{E}\left(I-\lambda_{n} B\right) x_{n}\right\|\right\},\right. \\
\left.\sup _{n \geq 1}\left\{\left\|P_{E}\left(I-\mu_{n} C\right) x_{n}\right\|\right\}, \sup _{n \geq 1}\left\{\left\|B x_{n}\right\|\right\}, \sup _{n \geq 1}\left\{\left\|C x_{n}\right\|\right\}, \sup _{n \geq 1}\left\{\left\|S_{k} x_{n}\right\|\right\}\right\} .
\end{gathered}
$$

From (C4), (C6), and (3.28), we obtain

$$
\lim _{n \rightarrow \infty}\left\|x_{n}-Q x_{n}\right\|=0
$$

Since $P_{\Theta}(I-A+\gamma f)(q)$ is a contraction with the coefficient $\alpha \in[0,1)$, we have that there exists a unique fixed point. We use $q$ to denote the unique fixed point to the mapping $P_{\Theta}(I-A+\gamma f)(q)$. That is, $q=P_{\Theta}(I-A+\gamma f)(q)$. Since $\left\{x_{n_{i}}\right\}$ is bounded, there exists a subsequence $\left\{x_{n_{i}}\right\}$ of $\left\{x_{n}\right\}$ 
which converges weakly to $z$. Without loss of generality, we may assume that $\left\{x_{n_{i}}\right\} \rightarrow z$. It follows from (3.58), that

$$
\lim _{n \rightarrow \infty}\left\|x_{n_{i}}-Q x_{n_{i}}\right\|=0
$$

It follows from Lemma 2.8 that $z \in F(\mathcal{Q})$. By (3.55), we have $z \in \Theta$.

Hence from (3.52) and (2.4), we arrive at

$$
\begin{aligned}
\limsup _{n \rightarrow \infty}\left\langle(A-\gamma f) q, q-x_{n}\right\rangle & =\limsup _{n \rightarrow \infty}\left\langle(A-\gamma f) q, q-x_{n_{i}}\right\rangle \\
& =\langle(A-\gamma f) q, q-z\rangle \leq 0 .
\end{aligned}
$$

On the other hand, we have

$$
\begin{aligned}
\left\langle(A-\gamma f) q, q-x_{n+1}\right\rangle & =\left\langle(A-\gamma f) q, x_{n}-x_{n+1}\right\rangle+\left\langle(A-\gamma f) q, q-x_{n}\right\rangle \\
& \leq\|(A-\gamma f) q\|\left\|x_{n}-x_{n+1}\right\|+\left\langle(A-\gamma f) q, q-x_{n}\right\rangle .
\end{aligned}
$$

From (3.25) and (3.60), we obtain that

$$
\limsup _{n \rightarrow \infty}\left\langle(A-r f) q, q-x_{n+1}\right\rangle \leq 0
$$

Step 5. We claim that $\lim _{n \rightarrow \infty}\left\|x_{n}-q\right\|=0$.

Indeed, by (3.2) and using Lemmas 2.6 and 2.14, we observe that

$$
\begin{aligned}
\left\|x_{n+1}-q\right\|^{2}= & \left\|\epsilon_{n} \gamma f\left(x_{n}\right)+\beta_{n} x_{n}+\left(\left(1-\beta_{n}\right) I-\epsilon_{n} A\right) k_{n}-q\right\|^{2} \\
= & \left\|\left(\left(1-\beta_{n}\right) I-\epsilon_{n} A\right)\left(k_{n}-q\right)+\beta_{n}\left(x_{n}-q\right)+\epsilon_{n}\left(\gamma f\left(x_{n}\right)-A q\right)\right\|^{2} \\
\leq & \left\|\left(1-\beta_{n}\right) \frac{\left(\left(1-\beta_{n}\right) I-\epsilon_{n} A\right)}{\left(1-\beta_{n}\right)}\left(k_{n}-q\right)+\beta_{n}\left(x_{n}-q\right)\right\|^{2} \\
& +2 \epsilon_{n}\left\langle\gamma f\left(x_{n}\right)-A q, x_{n+1}-q\right\rangle \\
\leq & \left(1-\beta_{n}\right)\left\|\frac{\left(\left(1-\beta_{n}\right) I-\epsilon_{n} A\right)}{1-\beta_{n}}\left(k_{n}-q\right)\right\|^{2}+\beta_{n}\left\|x_{n}-q\right\|^{2}
\end{aligned}
$$


Fixed Point Theory and Applications

$$
\begin{aligned}
& +2 \epsilon_{n} \gamma\left\langle f\left(x_{n}\right)-f(q), x_{n+1}-q\right\rangle+2 \epsilon_{n}\left\langle\gamma f(q)-A q, x_{n+1}-q\right\rangle \\
\leq & \left(1-\beta_{n}\right)\left\|\frac{\left(\left(1-\beta_{n}\right) I-\epsilon_{n} A\right)}{1-\beta_{n}}\left(k_{n}-q\right)\right\|^{2}+\beta_{n}\left\|x_{n}-q\right\|^{2} \\
& +2 \epsilon_{n} \gamma \alpha\left\|x_{n}-q\right\|\left\|x_{n+1}-q\right\|+2 \epsilon_{n}\left\langle\gamma f(q)-A q, x_{n+1}-q\right\rangle \\
\leq & \frac{\left\|\left(1-\beta_{n}\right) I-\epsilon_{n} A\right\|^{2}}{1-\beta_{n}}\left\|k_{n}-q\right\|^{2}+\beta_{n}\left\|x_{n}-q\right\|^{2} \\
& +\epsilon_{n} \gamma \alpha\left(\left\|x_{n}-q\right\|^{2}+\left\|x_{n+1}-q\right\|^{2}\right)+2 \epsilon_{n}\left\langle\gamma f(q)-A q, x_{n+1}-q\right\rangle \\
\leq & \frac{\left\|\left(1-\beta_{n}\right) I-\epsilon_{n} A\right\|^{2}}{1-\beta_{n}}\left\|x_{n}-q\right\|^{2}+\beta_{n}\left\|x_{n}-q\right\|^{2} \\
& +\epsilon_{n} \gamma \alpha\left(\left\|x_{n}-q\right\|^{2}+\left\|x_{n+1}-q\right\|^{2}\right)+2 \epsilon_{n}\left\langle\gamma f(q)-A q, x_{n+1}-q\right\rangle \\
\leq & \left(\frac{\left(\left(1-\beta_{n}\right)-\bar{\gamma} \epsilon_{n}\right)^{2}}{1-\beta_{n}}+\beta_{n}+\epsilon_{n} \gamma \alpha\right)\left\|x_{n}-q\right\|^{2} \\
& +\epsilon_{n} \gamma \alpha\left\|x_{n+1}-q\right\|^{2}+2 \epsilon_{n}\left\langle\gamma f(q)-A q, x_{n+1}-q\right\rangle \\
\leq & \left(1-(2 \bar{\gamma}-\alpha \gamma) \epsilon_{n}+\frac{\bar{\gamma}^{2} \epsilon_{n}^{2}}{1-\beta_{n}}\right)\left\|x_{n}-q\right\|^{2} \\
& +\epsilon_{n} \gamma \alpha\left\|x_{n+1}-q\right\|^{2}+2 \epsilon_{n}\left\langle\gamma f(q)-A q, x_{n+1}-q\right\rangle,
\end{aligned}
$$

which implies that

$$
\begin{aligned}
\left\|x_{n+1}-q\right\|^{2} \leq & \left(1-\frac{2(\bar{\gamma}-\alpha \gamma) \epsilon_{n}}{1-\alpha \gamma \epsilon_{n}}\right)\left\|x_{n}-q\right\|^{2} \\
& +\frac{\epsilon_{n}}{1-\alpha \gamma \epsilon_{n}}\left\{\frac{\bar{\gamma}^{2} \epsilon_{n}}{1-\beta_{n}}\left\|x_{n}-q\right\|^{2}+2\left\langle\gamma f(q)-A q, x_{n+1}-q\right\rangle\right\} .
\end{aligned}
$$

Taking

$$
\begin{gathered}
\sigma_{n}=\frac{\epsilon_{n}}{1-\alpha \gamma \epsilon_{n}}\left\{\frac{\bar{\gamma}^{2} \epsilon_{n}}{1-\beta_{n}}\left\|x_{n}-q\right\|^{2}+2\left\langle\gamma f(q)-A q, x_{n+1}-q\right\rangle\right\} \\
\rho_{n}=\frac{2(\bar{\gamma}-\alpha \gamma) \epsilon_{n}}{1-\alpha \gamma \epsilon_{n}}
\end{gathered}
$$

then, we can rewrite (3.64) as

$$
\left\|x_{n+1}-q\right\|^{2} \leq\left(1-Q_{n}\right)\left\|x_{n}-q\right\|^{2}+\sigma_{n},
$$


and we can see that $\sum_{n=1}^{\infty} \varphi_{n}=\infty$ and $\lim \sup _{n \rightarrow \infty}\left(\sigma_{n} / \varphi_{n}\right) \leq 0$. Applying Lemma 2.13 to (3.66), we conclude that $\left\{x_{n}\right\}$ converges strongly to $q$ in norm. This completes the proof.

If the mapping $S$ is nonexpansive, then $S_{k}=S_{0}=S$. We can obtain the following result from Theorem 3.1 immediately.

Corollary 3.2. Let $E$ be a nonempty closed convex subset of a real Hilbert space $H$. Let $\Phi_{1}$ and $\Phi_{2}$ be two bifunctions from $E \times E$ to $R$ satisfying (A1)-(A5) and let $\varphi: E \rightarrow \mathcal{R} \cup\{+\infty\}$ be a proper lower semicontinuous and convex function with either (B1) or (B2). Let $C: E \rightarrow H$ be a $\xi$-inverse-strongly monotone mapping, let $\Psi_{1}: E \rightarrow H$ be a $\rho$-inverse-strongly monotone mapping, let $\Psi_{2}: E \rightarrow H$ be an $\omega$-inverse-strongly monotone mapping and let $B: E \rightarrow H$ be a $\beta$-inverse-strongly monotone mapping. Let $f: E \rightarrow E$ be an $\alpha$-contraction with coefficient $\alpha(0 \leq \alpha<1)$ and let $A$ be a strongly positive linear bounded operator on $H$ with coefficient $\bar{\gamma}>0$ and $0<\gamma<\bar{\gamma} / \alpha$. Let $S: E \rightarrow E$ be a nonexpansive mapping with a fixed point. Suppose that

$$
\Theta:=F(S) \cap V I(E, B) \cap V I(E, C) \cap G M E P\left(\Phi_{1}, \varphi, \Psi_{1}\right) \cap \operatorname{GMEP}\left(\Phi_{2}, \varphi, \Psi_{2}\right) \neq \emptyset
$$

Let $\left\{x_{n}\right\}$ be a sequence generated by the following iterative algorithm (3.2), where $\left\{\epsilon_{n}\right\},\left\{\beta_{n}\right\},\left\{\gamma_{n}\right\}$, and $\left\{\alpha_{n}^{(i)}\right\}$ are sequences in $(0,1)$, where $i=1,2,3,4,5, r \in(0,2 \rho), s \in(0,2 \omega)$, and $\left\{\lambda_{n}\right\}$ and $\left\{\mu_{n}\right\}$ are positive sequences. Assume that the control sequences satisfy (C1)-(C6) in Theorem 3.1. Then, $\left\{x_{n}\right\}$ converges strongly to a point $q \in \Theta$ which is the unique solution of the variational inequality:

$$
\langle(A-\gamma f) q, x-q\rangle \geq 0, \quad \forall x \in \Theta .
$$

Equivalently, one has $q=P_{\Theta}(I-A+\gamma f)(q)$.

If $\varphi=0, \Psi_{1}=\Psi_{2}=0, A=I, \gamma \equiv 1$, and $\gamma_{n}=1-\epsilon_{n}-\beta_{n}$ in Theorem 3.1, then we can obtain the following result immediately.

Corollary 3.3. Let $E$ be a nonempty closed convex subset of a real Hilbert space $H$. Let $\Phi_{1}$ and $\Phi_{2}$ be two bifunctions from $E \times E$ to $R$ satisfying (A1)-(A4). Let $C: E \rightarrow H$ be a $\xi$-inverse-strongly monotone mapping and let $B: E \rightarrow H$ be a $\beta$-inverse-strongly monotone mapping. Let $f: E \rightarrow E$ be an $\alpha$-contraction with coefficient $\alpha(0 \leq \alpha<1)$ and let $A$ be a strongly positive linear bounded operator on $H$ with coefficient $\bar{\gamma}>0$ and $0<\gamma<\bar{\gamma} / \alpha$. Let $S: E \rightarrow E$ be a $k$-strict pseudocontraction with a fixed point. Define a mapping $S_{k}: E \rightarrow E$ by $S_{k} x=k x+(1-k) S x$, for all $x \in E$. Suppose that

$$
\Theta:=F(S) \cap V I(E, C) \cap V I(E, B) \cap E P\left(\Phi_{1}\right) \cap E P\left(\Phi_{2}\right) \neq \emptyset .
$$


Let $\left\{x_{n}\right\}$ be a sequence generated by the following iterative algorithm:

$$
\begin{gathered}
x_{1} \in E, \quad u_{n} \in E, \quad v_{n} \in E, \\
\Phi_{1}\left(u_{n}, u\right)+\frac{1}{r}\left\langle u-u_{n}, u_{n}-x_{n}\right\rangle \geq 0, \quad \forall u \in E, \\
\Phi_{2}\left(v_{n}, v\right)+\frac{1}{s}\left\langle v-v_{n}, v_{n}-x_{n}\right\rangle \geq 0, \quad \forall v \in E, \\
z_{n}=P_{E}\left(x_{n}-\mu_{n} C x_{n}\right), \\
y_{n}=P_{E}\left(x_{n}-\lambda_{n} B x_{n}\right), \\
k_{n}=\alpha_{n}^{(1)} S_{k} x_{n}+\alpha_{n}^{(2)} y_{n}+\alpha_{n}^{(3)} z_{n}+\alpha_{n}^{(4)} u_{n}+\alpha_{n}^{(5)} v_{n}, \\
x_{n+1}=\epsilon_{n} f\left(x_{n}\right)+\beta_{n} x_{n}+\gamma_{n} k_{n}, \quad \forall n \geq 1,
\end{gathered}
$$

where $\left\{\epsilon_{n}\right\},\left\{\beta_{n}\right\},\left\{\gamma_{n}\right\}$, and $\left\{\alpha_{n}^{(i)}\right\}$ are sequences in $(0,1)$, where $i=1,2,3,4,5, r \in(0, \infty)$, $s \in(0, \infty)$, and $\left\{\lambda_{n}\right\}$ and $\left\{\mu_{n}\right\}$ are positive sequences. Assume that the control sequences satisfy the condition (C1)-(C6) in Theorem 3.1 and $\epsilon_{n}+\beta_{n}+\gamma_{n}=1$. Then, $\left\{x_{n}\right\}$ converges strongly to a point $q \in \Theta$, where $q=P_{\Theta} f(q)$.

If $B=0, C=0$, and $\Phi_{1}\left(u_{n}, u\right)=\Phi_{1}\left(v_{n}, v\right)=0$ in Corollary 3.3, then $P_{E}=I$ and we get $u_{n}=y_{n}=x_{n}$ and $v_{n}=z_{n}=x_{n}$; hence we can obtain the following result immediately.

Corollary 3.4. Let E be a nonempty closed convex subset of a real Hilbert space $H$. Let $S: E \rightarrow E$ be a $k$-strict pseudocontraction with a fixed point. Define a mapping $S_{k}: E \rightarrow E$ by $S_{k} x=k x+(1-$ $k) S x$, for all $x \in E$. Suppose that $F(S) \neq \emptyset$. Let $\left\{x_{n}\right\}$ be a sequence generated by the following iterative algorithm:

$$
\begin{gathered}
x_{1} \in E, \\
k_{n}=\alpha_{n} S_{k} x_{n}+\left(1-\alpha_{n}\right) x_{n}, \\
x_{n+1}=\epsilon_{n} f\left(x_{n}\right)+\beta_{n} x_{n}+\gamma_{n} k_{n}, \quad \forall n \geq 1,
\end{gathered}
$$

where $\left\{\epsilon_{n}\right\},\left\{\beta_{n}\right\},\left\{\gamma_{n}\right\}$, and $\left\{\alpha_{n}\right\}$ are sequences in $(0,1)$. Assume that the control sequences satisfy the conditions (C2) and (C3), $\lim _{n \rightarrow \infty} \alpha_{n}=\alpha \in(0,1)$ in Theorem 3.1, and $\epsilon_{n}+\beta_{n}+\gamma_{n}=1$. Then, $\left\{x_{n}\right\}$ converges strongly to a point $q \in F(S)$, where $q=P_{F(S)} f(q)$.

Finally, we consider the following Convex Feasibility Problem (CFP):

$$
\text { finding an } x \in \bigcap_{i=1}^{M} C_{i} \text {, }
$$

where $M \geq 1$ is an integer and each $C_{i}$ is assumed to be the of solutions of equilibrium problem with the bifunction $\Phi_{i}, i=1,2,3, \ldots, M$ and the solution set of the variational inequality problem. There is a considerable investigation on CEP in the setting of Hilbert 
spaces which captures applications in various disciplines such as image restoration [38, 39], computer tomography [40], and radiation therapy treatment planning [41].

The following result can be obtained from Theorem 3.1. We, therefore, omit the proof.

Theorem 3.5. Let $E$ be a nonempty closed convex subset of a real Hilbert space $H$. Let be a $\Phi_{i}$ bifunction from $E \times E$ to $\mathcal{R}$ satisfying $(A 1)-(A 5)$ and let $\varphi: E \rightarrow \mathcal{R} \cup\{+\infty\}$ be a proper lower semicontinuous and convex function with either (B1) or (B2). Let $C_{i}: E \rightarrow H$ be an $\xi_{i}$-inversestrongly monotone mapping for each $i \in\{1,2,3, \ldots, N\}$. Let $f: E \rightarrow E$ be a contraction mapping with coefficient $\alpha(0<\alpha<1)$ and let $A$ be a strongly positive linear bounded operator on $H$ with coefficient $\bar{\gamma}>0$ and $0<\gamma<\bar{\gamma} / \alpha$. Let $S: E \rightarrow E$ be a $k$-strict pseudocontraction with a fixed point. Define a mapping $S_{k}: E \rightarrow E$ by $S_{k} x=k x+(1-k) S x$, for all $x \in E$. Suppose that

$$
\mathcal{F}:=F(S) \cap\left(\bigcap_{i=1}^{N} V I\left(E, C_{i}\right)\right) \cap\left(\bigcap_{j=1}^{M} \operatorname{MEP}\left(\Phi_{j}, \varphi, \Psi_{j}\right)\right) \neq \emptyset .
$$

Let $\left\{x_{n}\right\}$ be a sequence generated by the following iterative algorithm:

$$
\begin{gathered}
x_{1} \in E, \quad u_{M} \in E \\
\Phi_{1}\left(u_{n, N+1}, u_{1}\right)+\varphi(x)-\varphi\left(u_{n, N+1}\right)+\left\langle\Psi_{1} x_{n}, u-u_{n, N+1}\right\rangle+\frac{1}{r_{1}}\left\langle u_{1}-u_{n, N+1}, u_{n, N+1}-x_{n}\right\rangle \geq 0, \quad \forall u_{1} \in E, \\
\Phi_{2}\left(u_{n, N+2}, u_{2}\right)+\varphi(x)-\varphi\left(u_{n, N+2}\right)+\left\langle\Psi_{2} x_{n}, u-u_{n, N+2}\right\rangle+\frac{1}{r_{2}}\left\langle u_{2}-u_{n, N+2}, u_{n, N+2}-x_{n}\right\rangle \geq 0, \quad \forall u_{2} \in E, \\
\vdots \\
\Phi_{N}\left(u_{n, M}, u_{M}\right)+\varphi(x)-\varphi\left(u_{n, M}\right)+\left\langle\Psi_{M} x_{n}, u-u_{n, M}\right\rangle+\frac{1}{r_{N}}\left\langle u_{M}-u_{n, M}, u_{n, M}-x_{n}\right\rangle \geq 0, \quad \forall u_{M} \in E, \\
z_{n, 1}=P_{E}\left(x_{n}-\mu_{n, 1} C_{1} x_{n}\right), \\
z_{n, 2}=P_{E}\left(x_{n}-\mu_{n, 2} C_{2} x_{n}\right), \\
\vdots \\
z_{n, N}=P_{E}\left(x_{n}-\mu_{n, N} C_{N} x_{n}\right), \\
k_{n}=\alpha_{n, 0} S_{k} x_{n}+\sum_{i=1}^{N} \alpha_{n, i} z_{n, i}+\sum_{j=N+1}^{M} \alpha_{n, j} u_{n, j}, \\
x_{n+1}=\epsilon_{n} \gamma f\left(x_{n}\right)+\beta_{n} x_{n}+\left(\left(1-\beta_{n}\right) I-\epsilon_{n} A\right) k_{n}, \quad \forall n \geq 1,
\end{gathered}
$$

where $\alpha_{n, 0}, \alpha_{n, 1}, \alpha_{n, 2}, \alpha_{n, 3}, \alpha_{n, N}, \ldots, \alpha_{n, N+1}, \ldots, \alpha_{n, M} \in(0,1)$ such that $\sum_{i=0}^{M} \alpha_{n, i}=1,\left\{\mu_{n, i}\right\}$ are positive sequences, and $\left\{\epsilon_{n}\right\}$ and $\left\{\beta_{n}\right\}$ are sequences in $(0,1)$. Assume that the control sequences 
satisfy the following restrictions:

(C1) $\lim _{n \rightarrow \infty} \epsilon_{n}=0$ and $\sum_{n=1}^{\infty} \epsilon_{n}=\infty$,

(C2) $0<\liminf _{n \rightarrow \infty} \beta_{n} \leq \lim \sup _{n \rightarrow \infty} \beta_{n}<1$,

(C3) $\lim _{n \rightarrow \infty}\left|\mu_{n+1, i}-\mu_{n, i}\right|=0$, for each $1 \leq i \leq N$,

(C4) $e_{i} \leq \mu_{n, i} \leq 2 \xi_{i}$, where $e_{i}$ is some positive constant for each $1 \leq i \leq N$,

(C5) $\lim _{n \rightarrow \infty} \alpha_{n, i}=\alpha_{i} \in(0,1)$, for each $1 \leq i \leq M$.

Then, $\left\{x_{n}\right\}$ converges strongly to a point $q \in \mathcal{F}$ which is the unique solution of the variational inequality:

$$
\langle(A-\gamma f) q, x-q\rangle \geq 0, \quad \forall x \in \mathcal{F} .
$$

Equivalently, one has $q=P_{\bar{F}}(I-A+\gamma f)(q)$.

\section{Acknowledgments}

The authors are grateful to the anonymous referees for their helpful comments which improved the presentation of the original version of this paper. The first author was supported by the Thailand Research Fund and the Commission on Higher Education under Grant No. MRG5380044. The second author was supported by Rajamangala University of Technology Rattanakosin Research and Development Institute.

\section{References}

[1] W. Takahashi, Nonlinear Functional Analysis, Yokohama Publishers, Yokohama, Japan, 2000.

[2] F. E. Browder and W. V. Petryshyn, "Construction of fixed points of nonlinear mappings in Hilbert space," Journal of Mathematical Analysis and Applications, vol. 20, pp. 197-228, 1967.

[3] G. Marino and H.-K. Xu, "Weak and strong convergence theorems for strict pseudo-contractions in Hilbert spaces," Journal of Mathematical Analysis and Applications, vol. 329, no. 1, pp. 336-346, 2007.

[4] H. Zhou, "Convergence theorems of fixed points for $k$-strict pseudo-contractions in Hilbert spaces," Nonlinear Analysis, vol. 69, no. 2, pp. 456-462, 2008.

[5] L.-C. Ceng and J.-C. Yao, "A hybrid iterative scheme for mixed equilibrium problems and fixed point problems," Journal of Computational and Applied Mathematics, vol. 214, no. 1, pp. 186-201, 2008.

[6] W. Takahashi and M. Toyoda, "Weak convergence theorems for nonexpansive mappings and monotone mappings," Journal of Optimization Theory and Applications, vol. 118, no. 2, pp. 417-428, 2003.

[7] E. Blum and W. Oettli, "From optimization and variational inequalities to equilibrium problems," The Mathematics Student, vol. 63, no. 1-4, pp. 123-145, 1994.

[8] F. E. Browder, "Existence and approximation of solutions of nonlinear variational inequalities," Proceedings of the National Academy of Sciences of the United States of America, vol. 56, pp. 1080-1086, 1966.

[9] P. Hartman and G. Stampacchia, "On some nonlinear elliptic differential-functional equations," Acta Mathematica, vol. 115, pp. 271-310, 1966.

[10] J.-C. Yao and O. Chadli, "Pseudomonotone complementarity problems and variational inequalities," in Handbook of Generalized Convexity and Generalized Monotonicity, N. Haddjissas and S. Schaible, Eds., vol. 76, pp. 501-558, Springer, New York, NY, USA, 2005.

[11] L. C. Zeng, S. Schaible, and J. C. Yao, "Iterative algorithm for generalized set-valued strongly nonlinear mixed variational-like inequalities," Journal of Optimization Theory and Applications, vol. 124, no. 3, pp. 725-738, 2005. 
[12] P. L. Combettes and S. A. Hirstoaga, "Equilibrium programming using proximal-like algorithms," Mathematical Programming, vol. 78, no. 1, pp. 29-41, 1997.

[13] X. Qin, Y. J. Cho, and S. M. Kang, "Viscosity approximation methods for generalized equilibrium problems and fixed point problems with applications," Nonlinear Analysis, vol. 72, no. 1, pp. 99-112, 2010.

[14] X. Gao and Y. Guo, "Strong convergence of a modified iterative algorithm for mixed-equilibrium problems in Hilbert spaces," Journal of Inequalities and Applications, vol. 2008, Article ID 454181, 23 pages, 2008.

[15] C. Jaiboon and P. Kumam, "A hybrid extragradient viscosity approximation method for solving equilibrium problems and fixed point problems of infinitely many nonexpansive mappings," Fixed Point Theory and Applications, vol. 2009, Article ID 374815, 32 pages, 2009.

[16] C. Jaiboon and P. Kumam, "Strong convergence for generalized equilibrium problems, fixed point problems and relaxed cocoercive variational inequalities," Journal of Inequalities and Applications, vol. 2010, Article ID 728028, 43 pages, 2010.

[17] C. Jaiboon and P. Kumam, "A general iterative method for addressing mixed equilibrium problems and optimization problems," Nonlinear Analysis, vol. 73, no. 5, pp. 1180-1202, 2010.

[18] C. Jaiboon, P. Kumam, and U. W. Humphries, "Weak convergence theorem by an extragradient method for variational inequality, equilibrium and fixed point problems," Bulletin of the Malaysian Mathematical Sciences Society, vol. 32, no. 2, pp. 173-185, 2009.

[19] J. S. Jung, "Strong convergence of composite iterative methods for equilibrium problems and fixed point problems," Applied Mathematics and Computation, vol. 213, no. 2, pp. 498-505, 2009.

[20] P. Kumam and C. Jaiboon, "A new hybrid iterative method for mixed equilibrium problems and variational inequality problem for relaxed cocoercive mappings with application to optimization problems," Nonlinear Anal: Hybrid Systems, vol. 3, no. 4, pp. 510-530, 2009.

[21] J.-W. Peng and J.-C. Yao, "Strong convergence theorems of iterative scheme based on the extragradient method for mixed equilibrium problems and fixed point problems," Mathematical and Computer Modelling, vol. 49, no. 9-10, pp. 1816-1828, 2009.

[22] S. Takahashi and W. Takahashi, "Viscosity approximation methods for equilibrium problems and fixed point problems in Hilbert spaces," Journal of Mathematical Analysis and Applications, vol. 331, no. 1, pp. 506-515, 2007.

[23] Y. Yao, Y.-C. Liou, and J.-C. Yao, "A new hybrid iterative algorithm for fixed-point problems, variational inequality problems, and mixed equilibrium problems," Fixed Point Theory and Applications, vol. 2008, Article ID 417089, 15 pages, 2008.

[24] Y. Yao and J.-C. Yao, "On modified iterative method for nonexpansive mappings and monotone mappings," Applied Mathematics and Computation, vol. 186, no. 2, pp. 1551-1558, 2007.

[25] G. Marino and H.-K. Xu, "A general iterative method for nonexpansive mappings in Hilbert spaces," Journal of Mathematical Analysis and Applications, vol. 318, no. 1, pp. 43-52, 2006.

[26] S. Plubtieng and R. Punpaeng, "A general iterative method for equilibrium problems and fixed point problems in Hilbert spaces," Journal of Mathematical Analysis and Applications, vol. 336, no. 1, pp. 455469, 2007.

[27] L.-C. Ceng, S. A. Al-Homidan, Q. H. Ansari, and J.-C. Yao, “An iterative scheme for equilibrium problems and fixed point problems of strict pseudo-contraction mappings," Journal of Computational and Applied Mathematics, vol. 223, no. 2, pp. 967-974, 2009.

[28] Y. Liu, "A general iterative method for equilibrium problems and strict pseudo-contractions in Hilbert spaces," Nonlinear Analysis, vol. 71, no. 10, pp. 4852-4861, 2009.

[29] R. Wangkeeree and R. Wangkeeree, "A general iterative method for variational inequality problems, mixed equilibrium problems, and fixed point problems of strictly pseudocontractive mappings in Hilbert spaces," Fixed Point Theory and Applications, vol. 2009, Article ID 519065, 32 pages, 2009.

[30] X. Qin and S. M. Kang, "Convergence theorems on an iterative method for variational inequality problems and fixed point problems," Bulletin of the Malaysian Mathematical Sciences Society, vol. 33, no. 1, pp. 155-167, 2010.

[31] R. T. Rockafellar, "On the maximality of sums of nonlinear monotone operators," Transactions of the American Mathematical Society, vol. 149, pp. 75-88, 1970.

[32] M. O. Osilike and D. I. Igbokwe, "Weak and strong convergence theorems for fixed points of pseudocontractions and solutions of monotone type operator equations," Computers $\mathcal{E}$ Mathematics with Applications, vol. 40, no. 4-5, pp. 559-567, 2000. 
[33] F. E. Browder, "Nonlinear operators and nonlinear equations of evolution in Banach spaces," in Nonlinear functional analysis (Proc. Sympos. Pure Math., Vol. XVIII, Part 2, Chicago, Ill, 1968), pp. 1-308, American Mathematical Society, Providence, RI, USA, 1976.

[34] R. E. Bruck Jr., "Properties of fixed-point sets of nonexpansive mappings in Banach spaces," Transactions of the American Mathematical Society, vol. 179, pp. 251-262, 1973.

[35] J.-W. Peng and J.-C. Yao, “A new hybrid-extragradient method for generalized mixed equilibrium problems, fixed point problems and variational inequality problems," Taiwanese Journal of Mathematics, vol. 12, no. 6, pp. 1401-1432, 2008.

[36] T. Suzuki, "Strong convergence of Krasnoselskii and Mann's type sequences for one-parameter nonexpansive semigroups without Bochner integrals," Journal of Mathematical Analysis and Applications, vol. 305, no. 1, pp. 227-239, 2005.

[37] H.-K. Xu, "Viscosity approximation methods for nonexpansive mappings," Journal of Mathematical Analysis and Applications, vol. 298, no. 1, pp. 279-291, 2004.

[38] P. L. Combettes, "The convex feasibility problem: in image recovery," in Advances Imaging and Electron Physics, P. Hawkes, Ed., vol. 95, pp. 155-270, Academic Press, Orlando, Fla, USA, 1996.

[39] T. Kotzer, N. Cohen, and J. Shamir, "Images to ration by a novel method of parallel projection onto constraint sets," Optics Letters, vol. 20, pp. 1172-1174, 1995.

[40] M. I. Sezan and H. Stark, "Application of convex projection theory to image recovery in tomograph and related areas," in Image Recovery: Theory and Application, H. Stark, Ed., pp. 155-270, Academic Press, Orlando, Fla, USA, 1987.

[41] Y. Censor and S. A. Zenios, Parallel Optimization, Numerical Mathematics and Scientific Computation, Oxford University Press, New York, NY, USA, 1997. 